\title{
A DENSE SET OF OPERATORS WITH TINY COMMUTANTS
}

\author{
DOMINGO A. HERRERO
}

\begin{abstract}
For a (bounded linear) operator $T$ on a complex, separable, infinitedimensional Hilbert space $\mathscr{H}$, let $\mathscr{A}(T)$ and $\mathscr{A}^{a}(T)$ denote the weak closure of the polynomials in $T$ and, respectively, the weak closure of the rational functions with poles outside the spectrum of $T$. Let $\mathscr{A}^{\prime}(T)$ and $\mathscr{A}^{\prime \prime}(T)$ denote the commutant and, respectively, the double commutant of $T$. We say that $T$ has a tiny commutant if $\mathscr{A}^{\prime}(T)=\mathscr{A}^{a}(T)$. By constructing a large family of "models" and by using standard techniques of approximation, it is shown that $\{T \in \mathscr{L}(\mathscr{H}): T$ has a tiny commutant $\}$ is norm-dense in the algebra $\mathscr{L}(\mathscr{H})$ of all operators acting on $\mathscr{H}$. Other related results: Let Lat $\mathscr{B}$ denote the invariant subspace lattice of a subalgebra $\mathscr{B}$ of $\mathscr{L}(\mathscr{H})$. For a Jordan curve $\gamma \subset \mathbf{C}$, let $\hat{\gamma}$ denote the union of $\gamma$ and its interior; for $T \in \mathscr{L}(\mathscr{H})$, let $\rho_{s-F}(T)=\{\lambda \in \mathbf{C}: \lambda-T$ is a semi-Fredholm operator $\}$, and let $\rho_{s-F}^{+}(T)\left(\rho_{s-F}^{-}(T)\right)=\left\{\lambda \in \rho_{s-F}(T): \operatorname{ind}(\lambda-T)>0 \quad(<0\right.$, resp. $\left.)\right\}$. With this notation in mind, it is shown that $\left\{T \in \mathscr{L}(\mathscr{H}): \mathscr{A}(T)=\mathscr{A}^{a}(T)\right\}^{-}=$ $\left\{T \in \mathscr{L}(\mathscr{H}): \text { Lat } \mathscr{A}(T)=\text { Lat } \mathscr{A}^{a}(T)\right\}^{-}=\{A \in \mathscr{L}(\mathscr{H}):$ if $\gamma$ (Jordan curve) $\subset \rho_{s-F}^{ \pm}(A)$, then $\left.\hat{\gamma} \subset \sigma(A)\right\}$; moreover, $\{A \in \mathscr{L}(\mathscr{H}):$ if $\gamma$ (Jordan curve) $\subset \rho_{s-F}^{ \pm}(A)$, then $\operatorname{ind}(\lambda-A)$ is constant on $\left.\hat{\gamma} \cap \rho_{s-F}(A)\right\} \subset\{T \in$ $\left.\mathscr{L}(\mathscr{H}): \mathscr{A}(T)=\mathscr{A}^{\prime}(T)\right\}^{-} \subset\left\{T \in \mathscr{L}(\mathscr{H}):\right.$ Lat $\mathscr{A}(T)=$ Lat $\left.\mathscr{A}^{\prime}(T)\right\} \subset\{A \in$ $\mathscr{L}(\mathscr{H}):$ if $\gamma$ (Jordan curve) $\subset \rho_{s-F}^{ \pm}(A)$, then $\left.\hat{\gamma} \cap \rho_{s-F}(A) \subset \rho_{s-F}^{ \pm}(A)\right\} \subset$ $\left\{T \in \mathscr{L}(\mathscr{H}): \mathscr{A}(T)=\mathscr{A}^{a}(T)\right\}$. (The first and the last inclusions are proper.) The results also include a partial analysis of Lat $\mathscr{A}^{\prime \prime}(T)$.
\end{abstract}

\section{INTRODUCTION}

To each operator $T$ we can naturally associate four weakly closed subalgebras with identity; namely,

$$
\mathscr{A}(T)=\text { the weak closure of the polynomials in } T \text { and } 1 \text {, }
$$

Received by the editors August 3,1989. The contents of this article were presented to the Society in the Special Session on Operator Theory at Irvine, California, November 10-11, 1990.

1980 Mathematics Subject Classification (1985 Revision). Primary 41A65, 47A15, 47C05; Secondary 47A53, 47A55.

Key words and phrases. Hilbert space operator, commutant, double commutant, weak closure of the polynomials, weak closure of the rational functions with poles outside the spectrum, invariant subspace lattices, tiny commutants, semi-Fredholm domain, approximation of operators.

This research has been partially supported by a grant from the National Science Foundation. 
$\mathscr{A}^{a}(T)=$ the weak closure of the rational functions in $T$ with poles outside the spectrum, $\sigma(T)$, of $T$,

$\mathscr{A}^{\prime}(T)=\{A \in \mathscr{L}(\mathscr{H}): T A=A T\}=$ the commutant of $T$, and

$\mathscr{A}^{\prime \prime}(T)=\left\{B \in \mathscr{L}(\mathscr{H}): A B=B A\right.$ for all $\left.A \in \mathscr{A}^{\prime}(T)\right\}=$ the double commutant of $T$.

(Here $\mathscr{L}(\mathscr{H})$ denotes the algebra of all bounded linear operators acting on the complex, separable, infinite-dimensional Hilbert space $\mathscr{H}$.)

Clearly, $\mathscr{A}(T) \subset \mathscr{A}^{a}(T) \subset \mathscr{A}^{\prime \prime}(T) \subset \mathscr{A}^{\prime}(T)$, and the corresponding invariant subspace lattices satisfy the reverse inclusions,

$$
\operatorname{Lat} \mathscr{A}(T) \supset \operatorname{Lat} \mathscr{A}^{a}(T) \supset \operatorname{Lat} \mathscr{A}^{\prime \prime}(T) \supset \text { Lat } \mathscr{A}^{\prime}(T)
$$

$\left(\right.$ Lat $\mathscr{A}(T)=$ Lat $T$ is just the invariant subspace lattice of $T ;$ Lat $\mathscr{A}^{\prime}(T)$ is the lattice of hyperinvariant subspaces of $T$, also denoted by Hyperlat $T$.)

The main result of this article says that operators with "tiny" commutants, in the sense that $\mathscr{A}^{\prime}(T)=\mathscr{A}^{a}(T)$, form a norm-dense subset of $\mathscr{L}(\mathscr{H})$. Obviously, the same result is true if the condition " $\mathscr{A}^{\prime}(T)=\mathscr{A}^{a}(T)$ " is replaced by " $\mathscr{A}^{\prime \prime}(T)=\mathscr{A}^{a}(T)$," or "Lat $\mathscr{A}^{a}(T)=$ Hyperlat $T$," or any other weaker condition. (Indeed, the density of $\left\{T \in \mathscr{L}(\mathscr{H}): \mathscr{A}^{\prime \prime}(T)=\mathscr{A}^{\prime}(T)\right\}$ follows immediately from [10, Class (D), p. 109].)

On the other hand, an invariant subspace $\mathscr{M}$ of $T$ is invariant under $\mathscr{A}^{a}(T)$ if and only if $\sigma(T \mid \mathscr{M}) \subset \sigma(T)$ [17]. (By subspace we always mean a closed linear manifold of $\mathscr{H} ; T \mid \mathscr{M}$ denotes the restriction of $T$ to $\mathscr{M}$.)

By using this observation, it is possible to show that $\{T \in \mathscr{L}(\mathscr{H}): \mathscr{A}(T)=$ $\left.\mathscr{A}^{a}(T)\right\}$ and $\left\{T \in \mathscr{L}(\mathscr{H})\right.$ : Lat $T=$ Lat $\left.\mathscr{A}^{a}(T)\right\}$ have exactly the same (norm) closure, and this closure admits a very simple spectral description and it is nowhere dense in $\mathscr{L}(\mathscr{H})$ (see Theorem 4.1 below).

This article sprouted out of a question of John B. Conway (personal communication):

What is the closure of the set $\{T \in \mathscr{L}(\mathscr{H})$ : Lat $T=$ Hyperlat $T\}$ ?

Although unable to answer this question, the author shows that the closure of the set of operators $T$ with Lat $T=$ Hyperlat $T$ can be "sandwiched" between two nowhere dense closed subsets of $\mathscr{L}(\mathscr{H})$ (both having simple spectral descriptions). In particular, the closure of this set is strictly included in $\left\{T \in \mathscr{L}(\mathscr{H}): \text { Lat } T=\text { Lat } \mathscr{A}^{a}(T)\right\}^{-}$.

All the subsets of $\mathscr{L}(\mathscr{H})$ considered here are similarity-invariant, and therefore we can apply the approximation machinery developed in the monographs [2] and [11] (see also [14], [15]).

This article was written during an Informal Seminar on Operator Theory at the University of California at San Diego (Summer, 1989). The author wishes 
to thank Professors J. Agler, L. C. Chadwick, and J. W. Helton, and the Department of Mathematics of U.C.S.D. for their generous invitation.

\section{CONSTRUCTION OF MODELS}

The core of the article is the following highly technical result on the existence of a peculiar type of operators with tiny commutants. The applications of these "models" will be developed in $\S \S 3,4$, and 5 .

Proposition 2.1. Let $\mathbf{D}$ denote the open unit disk and let $\left\{\mathbf{D}\left(\alpha_{j}, r_{j}\right)\right\}_{j=1}^{k}$ be a finite collection of open disks with pairwise disjoint closures, such that $\mathbf{D}\left(\alpha_{j}, r_{j}\right)^{-}$ is included in $\mathbf{D}(k=1,2, \ldots, k ; \mathbf{D}(\alpha, r)=\{\lambda \in \mathbf{C}:|\lambda-\alpha|<r\}, r+|\alpha|<1)$. Let $\Omega=\mathbf{D} \backslash \bigcup_{j=1}^{k} \mathbf{D}\left(\alpha_{j}, r_{j}\right)^{-}$.

Given an index $m, 1 \leq m \leq \infty$, there exists $T$ in $\mathscr{L}(\mathscr{H})$ such that

(i) $\sigma(T)=\Omega^{-}$and $\sigma_{l e}(T)=\partial \Omega$;

(ii) $\operatorname{ker}(\lambda-T)=\{0\}$ and $\operatorname{dim} \operatorname{ker}(\lambda-T)^{*}=m$ for all $\lambda \in \Omega$;

(iii) $\lambda-T$ is a semi-Fredholm operator of index $-m$ for all $\lambda \in \Omega$, and

(iv) $\mathscr{A}^{a}(T)=\mathscr{A}^{\prime}(T)$ is isomorphic (but not necessarily isometric) with $H^{\infty}(\Omega)$.

Here $\sigma_{l e}(T)$ and $\sigma_{r e}(T)$ denote the left and, respectively, the right essential spectrum of $T ; \sigma_{l r e}(T)=\sigma_{l e}(T) \cap \sigma_{r e}(T)$ is the complement in $\mathbf{C}$ of the semi-Fredholm domain of $T, \rho_{s-F}(T)=\{\lambda \in \mathbf{C}: \lambda-T$ is a semi-Fredholm operator $\}$. The essential spectrum is $\sigma_{e}(T)=\sigma_{l e}(T) \cup \sigma_{r e}(T)$.

Proposition 2.2. Let $\left\{\alpha_{j}\right\}_{j=1}^{k}$ be a finite subset of the open unit disk, and let $\boldsymbol{\Omega}=\mathbf{D} \backslash\left\{\alpha_{j}\right\}_{j=1}^{k}$.

Given an index $m, 1 \leq m \leq \infty$, there exists $T$ in $\mathscr{L}(\mathscr{H})$ such that

(i) $\sigma(T)=\mathbf{D}^{-}$and $\sigma_{l e}(T)=\partial \mathbf{D} \cup\left\{\alpha_{j}\right\}_{j=1}^{k}(=\partial \Omega)$;

(ii) $\operatorname{ker}(\lambda-T)=\{0\}$ and $\operatorname{dim} \operatorname{ker}(\lambda-T)^{*}=m$ for all $\lambda \in \Omega$;

(iii) $\lambda-T$ is a semi-Fredholm operator of index $-m$ for all $\lambda \in \Omega$, and

(iv) $\mathscr{A}(T)=\mathscr{A}^{\prime}(T)$ is isometrically isomorphic with $H^{\infty}(\mathbf{D})$.

The reader is referred to [4], [19] for definition and properties of the semiFredholm operators, and to treatise of B. Sz.-Nagy and C. Foias [23] for the definition and properties of the $H^{\infty}(\mathbf{D})$ functional calculus for contractions.

Our first result improves upon an example due to W. R. Wogen. In [25], Wogen proved that for each cardinal $m, 1 \leq m \leq \infty$, there is an operator $A$ in $\mathscr{L}(\mathscr{H})$ such that $\mathscr{A}^{\prime}(A)$ has multiplicity $m ; A$ is a particular type of unilateral operator weighted shift: Let $\mathscr{R}$ be a Hilbert space of dimension $m$, and let $\left\{g_{k}\right\}_{k=1}^{\infty}$ be a denumerable dense subset of the unit sphere of $\mathscr{R}$.

Define $R_{k}=\left(1-P_{k}\right)+(1 / k) P_{k}$, where $P_{k}$ denotes the orthogonal projection of $\mathscr{R}$ onto the one-dimensional space spanned by $g_{k}, k=1,2, \ldots$. 
Wogen proved that if

$$
T=\left(\begin{array}{cccccccc}
0 & & & & & & \\
T_{0} & 0 & & & & \\
& T_{1} & 0 & & 0 & \\
& & T_{2} & 0 & & & \\
& & & \cdot & . & \\
& & 0 & & . & . \\
& & & & & . & .
\end{array}\right) \begin{gathered}
\mathscr{R} \\
\mathscr{R} \\
\mathscr{R} \\
\mathscr{R} \\
\cdot \\
. \\
.
\end{gathered}
$$

where $T_{0}=R_{1}, T_{k}=(1 / k) R_{k+1} R_{k}^{-1}(k \geq 1)$, then every operator $A$ commuting with $T$ has a lower triangular operator matrix (with respect to the above decomposition) with diagonal terms $A_{00}=A_{11}=A_{22}=\cdots=\lambda_{0} 1_{\mathscr{R}}$ for some $\lambda_{0} \in C$. (From this we can immediately deduce that $\mathscr{A}^{\prime}(T)$ has multiplicity equal to $m$.)

A lot more can be said. (Beware, the calculations that follow are not for the feeble hearted reader!)

Lemma 2.3. Let $\left\{T_{n}\right\}_{n=0}^{\infty}$ be a bounded sequence of invertible operators in $\mathscr{R}$ such that for some subsequence $\left\{n_{k}\right\}_{k=1}^{\infty}$ and positive constants $\left\{\delta_{k}\right\}_{k=1}^{\infty}$ satisfies $T_{n_{k}} T_{n_{k}-1} \cdots T_{2} T_{1} T_{0}=\delta_{k} R_{k} \quad\left(k=1,2, \ldots ; R_{k}\right.$ is defined as above $)$. Assume, moreover, that $\left\|T_{n}^{-1}\right\| \leq M$ for all $n=0,1,2, \ldots$.

Let $T \in \mathscr{L}(\mathscr{H})$ be the unilateral operator weighted shift with weight sequence $\left\{T_{n}\right\}_{n=0}^{\infty}$ (defined by

$$
T=\left(\begin{array}{cccccccc}
0 & & & & & & \\
T_{0} & 0 & & & & & \\
& T_{1} & 0 & & 0 & \\
& \cdot & T_{2} & 0 & & \\
& & & \cdot & \cdot & \\
& 0 & & & \cdot & . \\
\mathscr{R} \\
\mathscr{R} \\
\mathscr{R} \\
\cdot \\
\cdot
\end{array}\right.
$$

with respect to the orthogonal direct sum decomposition $\mathscr{H}=\sum \bigoplus_{n=0}^{\infty} \mathscr{R}_{n}$, $\mathscr{R}_{n} \simeq \mathscr{R}$ for all $\left.n=0,1,2, \ldots\right)$.

Then $\mathscr{A}^{\prime}(T)=\mathscr{A}(T)$.

Proof. Let $A=\left(A_{i j}\right)_{i, j=0}^{\infty}$ be the matrix of $A \in \mathscr{A}^{\prime}(T)$ with respect to the given decomposition; then

$$
\begin{aligned}
O & =T A-A T \\
& =\left(\begin{array}{cccc}
-A_{01} T_{0} & -A_{02} T_{1} & -A_{03} T_{2} & \ldots \\
T_{0} A_{00}-A_{11} T_{0} & T_{0} A_{01}-A_{12} T_{1} & T_{0} A_{02}-A_{13} T_{2} & \ldots \\
T_{1} A_{10}-A_{20} T_{0} & T_{1} A_{11}-A_{22} T_{1} & T_{1} A_{12}-A_{23} T_{2} & \ldots \\
T_{2} A_{20}-A_{30} T_{0} & T_{2} A_{21}-A_{32} T_{1} & T_{2} A_{22}-A_{33} T_{2} & \ldots \\
\vdots & \vdots & \vdots & \ddots
\end{array}\right) .
\end{aligned}
$$

Since the $T_{n}$ 's are invertible, the first row indicates that $A_{0 n}=0$ for all $n \geq 1$. Now the second row indicates that $A_{1 n}=0$ for all $n \geq 2$, etc., so 
that $A$ has a lower triangular operator matrix. (This can also be deduced by observing that

$$
\mathscr{R}_{0}=\operatorname{ker} T^{*}, \quad \mathscr{R}_{0} \oplus \mathscr{R}_{1}=\operatorname{ker} T^{* 2}, \quad \mathscr{R}_{0} \oplus \mathscr{R}_{1} \oplus \mathscr{R}_{2}=\operatorname{ker} T^{* 3}, \ldots,
$$

are hyperinvariant subspaces of $T^{*}$, and therefore their orthogonal complements, $\sum \bigoplus_{n=1}^{\infty} \mathscr{R}_{n}, \sum_{n=2}^{\infty} \bigoplus \mathscr{R}_{n}, \sum \bigoplus_{n=3}^{\infty} \mathscr{R}_{n}, \ldots$ must be invariant under every $A$ in $\mathscr{A}^{\prime}(T)$.)

From the entries $(2,1),(3,2), \ldots,(n+1, n), \ldots$, we inductively obtain

$$
A_{n n}=\left(T_{n-1} T_{n-2} \cdots T_{0}\right) A_{00}\left(T_{n-1} T_{n-2} \cdots T_{0}\right)^{-1} \quad(n=1,2, \ldots) .
$$

Thus (as in Wogen's article [25]),

$$
\|A\| \geq\left\|A_{n_{k}+1, n_{k}+1}\right\|=\sup _{x \neq 0} \frac{\left\|\left(T_{n_{k}} T_{n_{k}-1} \ldots T_{0}\right) A_{00} x\right\|}{\left\|T_{n_{k}} T_{n_{k}-1} \ldots T_{0} x\right\|}=\sup _{x \neq 0} \frac{\left\|R_{k}\left(A_{00} x\right)\right\|}{\left\|R_{k} x\right\|} .
$$

If $A_{00}$ is not a multiple of the identity, then there is a unit vector $x_{0}$ such that $y_{0}=A_{00} x_{0}$ is linearly independent of $x_{0}$. By taking a subsequence $\left\{n_{k(i)}\right\}_{i=1}^{\infty}$ so that $\left\|x-g_{n_{k(i)}}\right\| \rightarrow 0 \quad(i \rightarrow \infty)$, we deduce that

$$
\|A\| \geq \sup _{i}\left\|A_{n_{k(i)}+1, n_{k(i)}+1} x_{0}\right\|=\sup _{i} \frac{\left\|R_{k(i)} y_{0}\right\|}{\left\|R_{k(i)} x_{0}\right\|}=\infty,
$$

a contradiction.

Hence, $A_{00}=\lambda_{0} 1_{\mathscr{R}}$, and therefore $A_{n n}=\lambda_{0} 1_{\mathscr{R}}$ for all $n \geq 0$ (for some $\left.\lambda_{0} \in \mathbf{C}\right)$.

Now consider the $(k+i, k)$-entries $T_{k+i-1} A_{k+i-1, k}-A_{k+i, k+1} T_{k}=0 \quad(i \geq$ $2, k \geq 0)$; equivalently, $T_{k+i} A_{k+i, k}=A_{k+i+1, k+1} T_{k}(i \geq 1, k \geq 0)$.

Fix $i$. By induction over $k$, we obtain

$$
\begin{aligned}
A_{n+i, n} & =\left(T_{n+i-1} T_{n+i-2} \cdots T_{i}\right) A_{i 0}\left(T_{n-1} T_{n-2} \cdots T_{0}\right)^{-1} \\
& =\left(T_{n+i-1} T_{n+i-2} \cdots T_{0}\right) S_{i 0}\left(T_{n-1} T_{n-2} \cdots T_{0}\right)^{-1},
\end{aligned}
$$

where $S_{i 0}=\left(T_{i-1} T_{i-2} \cdots T_{0}\right)^{-1} A_{i 0}$.

Thus,

$$
\begin{aligned}
\left\|A_{n+i, n}\right\| & =\sup _{x \neq 0} \frac{\left\|\left(T_{n+i-1} T_{n+i-2} \cdots T_{0}\right) S_{i 0} x\right\|}{\left\|T_{n-1} T_{n-2} \cdots T_{0} x\right\|} \\
& =\sup _{x \neq 0} \frac{\left\|\left(T_{n+i-1} T_{n+i-2} \cdots T_{n}\right)\left(T_{n-1} T_{n-2} \cdots T_{0}\right) S_{i 0} x\right\|}{\left\|T_{n-1} T_{n-2} \cdots T_{0} x\right\|} \\
& \geq M^{-i} \sup _{x \neq 0} \frac{\left\|\left(T_{n-1} T_{n-2} \cdots T_{0}\right) S_{i 0} x\right\|}{\left\|T_{n-1} T_{n-2} \cdots T_{0} x\right\|},
\end{aligned}
$$

whence we deduce (exactly as in the case of $A_{00}$ ) that $S_{i 0}=\lambda_{i} 1_{\mathscr{R}}$ for some $\lambda_{i} \in \mathrm{C}$; equivalently, $A_{i 0}=\lambda_{i} T_{i-1} T_{i-2} \cdots T_{0}$.

A fortiori, $A_{n+i, n}=\lambda_{i} T_{n+i-1} T_{n+i-2} \cdots T_{n}(i, n \geq 0)$. 
Observe that $T^{i}=\left(L_{h k}\right)_{h, k=0}^{\infty}$, where $L_{n+i, n}=T_{n+i-1} T_{n+i-2} \cdots T_{n} \quad(n \geq 0)$ and $L_{h k}=0$ if $h-k \neq i$. Hence, we can formally write $A=\sum_{i=0}^{\infty} \lambda_{i} T^{i}$.

Now it follows exactly as in the case of a unilateral scalar weighted shift that

$$
A=\text { (strong) } \lim _{m \rightarrow \infty} \sum_{i=0}^{m}\left(1-\frac{i}{m+1}\right) \lambda_{i} T^{i}
$$

(see [6], [22]).

Therefore, $A \in \mathscr{A}(T)$.

Remarks 2.4. (i) As mentioned before, W. R. Wogen considered the sequence $T_{0}=R_{1}, T_{n}=(1 / n) R_{n+1} R_{n}^{-1} \quad(n \geq 1)$. Since $R_{k}$ is a positive invertible operator, for each $m_{k} \geq 1$, we can write $R_{k}=\left(L_{k}\right)^{m_{k}}$, where $L_{k}=\left(1-P_{k}\right)+$ $(1 / k)^{1 / m_{k}} P_{k} \quad(k \geq 1)$. Clearly, if $m_{k}$ is large, then $1_{\mathscr{R}}-L_{k}$ is an operator of very small norm and rank equal to $m_{k}$.

Given a normed ideal $\mathscr{I}$ of compact operators, strictly larger than the trace class (see [19], [21]), we define

$$
\begin{aligned}
& T_{0}=1_{\mathscr{R}}, T_{1}=T_{2}=\cdots=T_{m_{1}}=L_{1}, T_{m_{1}+1}=T_{m_{1}+2}=\cdots=T_{3 m_{1}}=L_{1}^{-1}, \\
& T_{3 m_{1}+1}=T_{3 m_{1}+2}=\cdots=T_{4 m_{1}}=L_{1} \text {, } \\
& T_{4\left(m_{1}+\cdots+m_{k}\right)+1}=\cdots=T_{4\left(m_{1}+\cdots+m_{k}\right)+m_{k+1}}=L_{k+1}, \\
& T_{4\left(m_{1}+\cdots+m_{k}\right)+m_{k+1}+1}=\cdots=T_{4\left(m_{1}+\cdots+m_{k}\right)+3 m_{k+1}}=L_{k+1}^{-1} \text {, } \\
& T_{4\left(m_{1}+\cdots+m_{k}\right)+3 m_{k+1}+1}=\cdots=T_{4\left(m_{1}+\cdots+m_{k+1}\right)} \stackrel{k+1}{=} L_{k+1} \text {, }
\end{aligned}
$$

Let $T$ be defined as in Lemma 2.3 and let $S$ be the similarly defined operator with weights equal to $1_{\mathscr{R}}$ for all $n=0,1,2, \ldots S$ is the unilateral shift of multiplicity equal to $\operatorname{dim} \mathscr{R}$. Given $\varepsilon>0$, if $m_{k} \rightarrow \infty$ fast enough, then $S-T \in \mathscr{I}$ and $|S-T|_{\mathscr{I}}<\varepsilon$. In particular, $\sigma(T)=\mathbf{D}^{-}, \sigma_{l e}(T)=\partial \mathbf{D}$, and for each $\lambda$ in $\mathbf{D}, \lambda-T$ is a semi-Fredholm operator with index $-\operatorname{dim} \mathscr{R}$ and trivial kernel. Moreover, both $\left\{T_{n}\right\}_{n=0}^{\infty}$ and $\left\{T_{n}^{-1}\right\}_{n=0}^{\infty}$ have the property of the lemma. (This solves Proposition 2.1 for the case of a "nonperforated disk.")

(ii) If we define $T_{n}$ as above whenever $T_{n}=L_{k}$ (for some $k$ ) and $T_{n}=$ $\left(1 /\left\|L_{k}^{-1}\right\|\right) L_{k}$ whenever $T_{n}=L_{k}^{-1}$ (for some $k$ ), then $\|T\|=1$. It is easy to see that $T^{* k} \rightarrow 0$ (strongly, $k \rightarrow \infty$ ) and a simple calculation (by using the facts that $L_{k}^{m_{k}}=R_{k}$ and $\left\{g_{k}\right\}_{k=1}^{\infty}$ is dense in the unit sphere of $\mathscr{R}$ ) shows that $T^{k} \rightarrow 0$ (strongly, $k \rightarrow \infty$ ).

Thus, in this case $T$ is a $C_{00}$-contraction in the sense of B. Sz.-Nagy and C. Foias [23]; moreover, $T$ is very close to the shift $S$ of multiplicity $\operatorname{dim} \mathscr{R}$ (if $\mathscr{R}$ is finite-dimensional, $\left.|S-T|_{\mathscr{I}}<\varepsilon\right), \sigma(T)=\mathbf{D}, \sigma_{l e}(T)=\mathbf{D}$, and for each $\lambda \in \mathbf{D}, \lambda-T$ is a semi-Fredholm operator with index equal to $-\operatorname{dim} \mathscr{R}$ and trivial kernel. For suitable subsequences, $T_{n_{k}} T_{n_{k}-1} \cdots T_{1} T_{0}=\delta_{k} \mathscr{R}_{k}$ and $\left(T_{p_{k}} T_{p_{k}-1} \cdots T_{1} T_{0}\right)^{-1}=\eta_{k} R_{k}\left(\delta_{k}, \eta_{k}>0\right)$. Furthermore, if $\varphi \in H^{\infty}(\mathbf{D})$, 
$\varphi(\lambda)=\sum_{n=0}^{\infty} a_{n} \lambda^{n}$, then the mapping

$$
\varphi \rightarrow \varphi(T):=\left(\text { strong) } \lim _{m \rightarrow \infty} \varphi(r T)=(\text { strong }) \lim _{m \rightarrow \infty} \sum_{n=0}^{\infty} a_{n} r^{n} T^{n}\right.
$$

(these limits are well-defined) defines an isometric isomorphism from $H^{\infty}(\mathbf{D})$ onto $\mathscr{A}(T)$.

(iii) A close look of the proof of Lemma 2.3 shows that the condition " $\left\|T_{n}\right\| \leq$ $M$ for all $n \geq 0$ " is superabundant. The only thing we must guarantee is that $\left\|\left(T_{n+i-1} T_{n+i-2} \cdots T_{n}\right)^{-1}\right\|$ does not grow too fast. For instance, if we take $T_{0}=R_{1}, T_{1}=R_{2} R_{1}^{-1}$, and $T_{n}=(1 / n \log n) R_{n+1} R_{n}^{-1}$ for $n \geq 2$, then the proof of Lemma 2.3 yields a quasinilpotent unilateral operator weighted shift $T$ such that $\operatorname{dim} \operatorname{ker} T^{*}=\operatorname{dim} \mathscr{R}$ and $\mathscr{A}^{\prime}(T)=\mathscr{A}(T)$; moreover, if $\mathscr{R}$ is finite-dimensional, then $T$ is a compact operator.

As a second step toward the proof of Proposition 2.1, we shall settle the case of a single hole.

Lemma 2.5. Let $\mathbf{D}$ denote the open unit disk, let $\alpha \in \mathbf{D}$, and let $0<r<1-|\alpha|$ (so that $\left.\mathbf{D}(\alpha, r)^{-} \subset \mathbf{D}\right)$.

Define $\left\{T_{n}\right\}_{n=0}^{\infty}$ as in Remark 2.4(ii), and let $T \in \mathscr{L}(\mathscr{H})\left(\mathscr{H}=\sum \bigoplus_{n=-\infty}^{+\infty} \mathscr{R}_{n}\right.$, $\mathscr{R}_{n} \simeq \mathscr{R}$ ) be the operator defined by

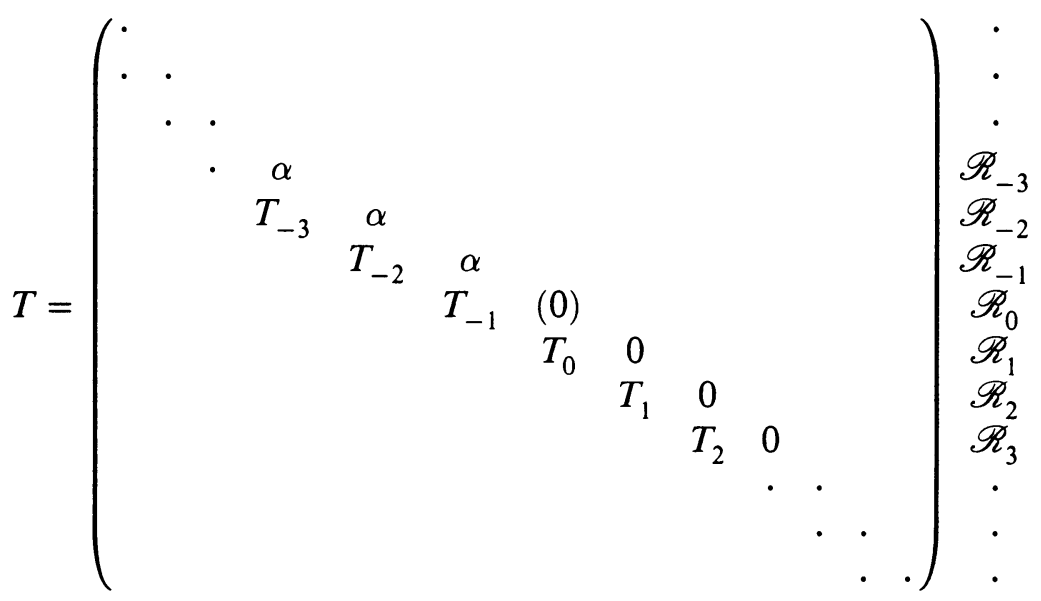

(the remaining entries of the matrix are 0 's), where $T_{-n}=r T_{n}^{-1}$.

Then $\sigma(T)=\mathbf{D}^{-} \backslash \mathbf{D}(\alpha, r), \quad T \mid \sum \bigoplus_{n=0}^{\infty} \mathscr{R}_{n}$ and $r(T-\alpha 1)^{-1} \mid \sum \bigoplus_{n=1}^{\infty} \mathscr{R}_{n}$ are $C_{00}$-contractions with $H^{\infty}(\mathbf{D})$ functional calculus,

$$
\mathscr{A}^{\prime}(T)=\mathscr{A}(T)=\left\{\varphi(T)+\psi\left(r(T-\alpha 1)^{-1}\right): \varphi, \psi \in H^{\infty}(\mathbf{D}), \psi(0)=0\right\}
$$

is isomorphic (although not necessarily isometric) with $H^{\infty}\left(\mathbf{D} \backslash \mathbf{D}(\alpha, r)^{-}\right)$; moreover, $\sigma_{l e}(T)=\partial \sigma(T)$ and, for each $\lambda$ in $\sigma(T) \backslash \partial \sigma(T), \lambda-T$ is a semiFredholm operator of index equal to $-\operatorname{dim} \mathscr{R}$ and trivial kernel. 
Proof. Let $A=\left(A_{i j}\right)_{-\infty}^{+\infty}$ be the matrix of $A \in \mathscr{A}^{\prime}(T)$ with respect to the given decomposition; then

$$
\begin{aligned}
0 & =T A-A T \\
= & \left(\begin{array}{c}
\left(T_{i-1} A_{i-1, j}-A_{i, j+1} T_{j}\right)_{i, j<0} \\
\left(T_{i-1} A_{i-1, j}-A_{i, j+1} T_{j}-\alpha A_{i j}\right)_{i \geq 0, j<0} \\
\left(T_{i-1} A_{i-1, j}-A_{i, j+1} T_{j}+\alpha A_{i j}\right)_{i<0, j \geq 0} \\
\left(T_{i-1} A_{i-1, j}-A_{i, j+1} T_{j}\right)_{i, j \geq 0}
\end{array}\right)
\end{aligned}
$$

with respect to the decomposition $\mathscr{H}=\left(\sum \bigoplus_{n<0} \mathscr{R}_{n}\right) \oplus\left(\sum \bigoplus_{n \geq 0} \mathscr{R}_{n}\right) \quad\left(\mathscr{H}_{1}=\right.$ $\left.\sum \bigoplus_{n<0} \mathscr{R}_{n}, \mathscr{H}_{0}=\sum \bigoplus_{n \geq 0} \mathscr{R}_{n}\right)$. By repeating four times the same arguments as in the proof of Lemma 2.3 , from the $(1,1)$-entry and the $(0,0)$-entry of the above $2 \times 2$ operator matrix, we deduce that

(1) $A_{i i}=a_{0} 1_{\mathscr{R}}$ for all $i \geq 0$,

(2) if $i, j \geq 0, i \neq j$, then either $A_{i j}=a_{i-j} T_{i-1} T_{i-2} \cdots T_{j}$ (for $i>j$ ), or $A_{i j}=a_{-(j-i)}\left(T_{j-1} T_{j-2} \cdots T_{i}\right)^{-1}$ (for $\left.i<j\right)$,

(3) $A_{i i}=b_{0} 1_{\mathscr{R}}$ for all $i<0$,

(4) if $i, j<0, i \neq j$, then either $A_{i j}=b_{i-j} T_{i-1} T_{i-2} \cdots T_{j}$ (for $i>j$ ), or $A_{i j}=b_{-(j-i)}\left(T_{j-1} T_{j-2} \cdots T_{i}\right)^{-1}$ (for $\left.i<j\right)$, where $a_{n}, b_{n} \in \mathbf{C} \quad(n \in$ Z), $a_{-1}=b_{-1}$, and $\lim \sup _{n \rightarrow \infty}\left|a_{n}\right|^{1 / n} \leq 1, \lim \sup _{n \rightarrow \infty}\left|b_{-n}\right|^{1 / n} \leq r$ (because the entries of $A$ are uniformly bounded by $\|A\|$ ); moreover, we also have $A_{i 0}=b_{i}\left(T_{-1} T_{-2} \cdots T_{-i}\right)^{-1}$ for all $i<0$.

On the other hand, the analysis of the $(0,1)$-entry of $T A-A T=0$ indicates that

(5) if $i \geq 0>j$, then $A_{i j}=\alpha^{-1}\left(T_{i-1} A_{i-1, j}-A_{i, j+1} T_{j}\right)$ is uniquely determined by $A_{i-1, j}$ (=the entry exactly above $A_{i j}$ in the matrix) and $A_{i, j+1}$ (=the entry to the right of $A_{i j}$ in the matrix), and a straightforward computation shows that $A_{i j}=c_{i j} T_{i-1} T_{i-2} \cdots T_{j}$.

Similarly, the analysis of the $(1,0)$-entry of $T A-A T=0$ indicates that

(6) if $i<0 \leq j$, then $A_{i, j+1}=\left(T_{i-1} A_{i-1, j}+\alpha A_{i j}\right) T_{j}^{-1}$; equivalently, if $i<0<j$, then $A_{i j}=\left(T_{i-1} A_{i-1, j-1}+\alpha A_{i, j-1}\right) T_{j-1}^{-1}$ is uniquely determined by $A_{i-1, j-1}$ (=the entry above and to the left of $A_{i j}$ in the matrix), and $A_{i, j-1}$ (=the entry to the left of $A_{i j}$ in the matrix), and a straightforward computation shows that $A_{i j}=c_{i j}\left(T_{j-1} T_{j-2} \cdots T_{i}\right)^{-1}$.

In both (5) and (6), $c_{i j} \in \mathbf{C}$ is defined by certain linear combinations of finitely many $a_{n}$ 's and $b_{n}$ 's.

Since $T=\left(\begin{array}{cc}T(1,1) & 0 \\ T(0,1) & T(0,0)\end{array}\right)$, where $T(0,0)$ has the form of Lemma 2.3 and $T(1,1)-\alpha$ is the adjoint of an operator of the form of Lemma 2.3, $\sigma(T(0,0))$ $=\mathbf{D}^{-}$and $\sigma(T(1,1)-\alpha)=r \mathbf{D}^{-}$, it is easy to see that $\sigma(T) \subset \mathbf{D}^{-}$and $T-\lambda$ is a semi-Fredholm operator with index equal to $-\operatorname{dim} \mathscr{R}$ and trivial kernel for all $\lambda \in \mathbf{D} \backslash \mathbf{D}(\alpha, r)^{-}$. 
On the other hand, by solving the equation $A(T-\alpha)=1$ ( $A$ as above) we can check that $T-\alpha$ is invertible, with inverse

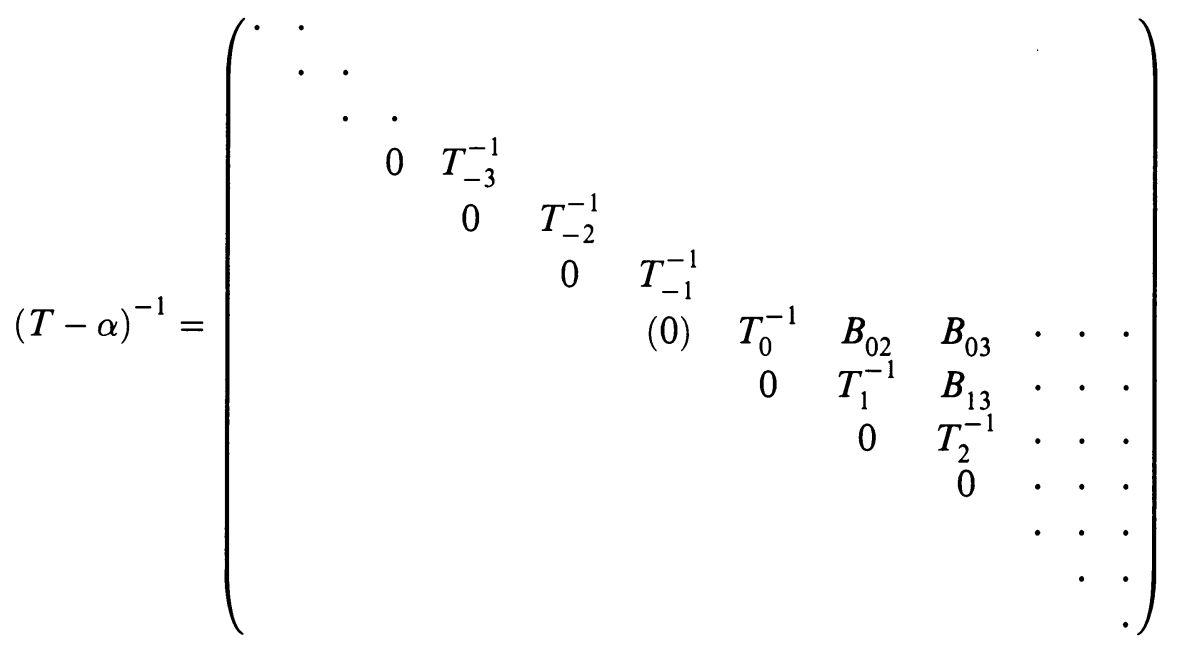

(the remaining entries of the matrix are 0's), where

$$
B_{i j}=\alpha^{j-i-1}\left(T_{j-1} T_{j-2} \cdots T_{i}\right)^{-1} \quad(j-1>i \geq 0)
$$

A straightforward computation shows that

$$
\begin{aligned}
\left\|(T-\alpha)^{-1}\right\| & \leq \max \left[r^{-1}, \sum_{n=0}^{\infty}((1+\eta)|\alpha|)^{n}\right] \\
& =\max \left[r^{-1},(1-(1+\eta)|\alpha|)^{-1}\right]=r^{-1},
\end{aligned}
$$

where $\eta=\max _{n \geq 0}\left(\left\|T_{n}^{-1}\right\|-1\right)$ satisfies $1-(1+\eta)|\alpha|>r$, provided all the $m_{k}$ 's of Remark 2.4 are sufficiently large.

Since we obviously have $\partial \mathbf{D}(\alpha, r) \subset \sigma(T)$, it follows that $\left\|(T-\alpha)^{-1}\right\|^{-1}=$ $r=\operatorname{dist}[\alpha, \partial \mathbf{D}(\alpha, r)]$. Therefore, $T-\lambda$ is invertible for all $\lambda \in \mathbf{D}(\alpha, r)$ $\left(\left\|(T-\lambda)^{-1}\right\|=(\operatorname{dist}[\lambda, \partial \mathbf{D}(\alpha, r)])^{-1}=(1-|\lambda-\alpha|)^{-1}\right)$, and $\left\|r(T-\alpha)^{-1}\right\|=1$; that is, $r(T-\alpha)^{-1}$ is a contraction.

Thus, $\sigma(T)=\mathbf{D}^{-} \backslash \mathbf{D}(\alpha, r)$ and $\sigma_{l e}(T)=\partial \mathbf{D} \cup \partial \mathbf{D}(\alpha, r)$. By using their matricial representations, it is not difficult to check that both $T$ and $r(T-$ $\alpha)^{-1}$ are $C_{00}$-contractions, and therefore they admit $H^{\infty}(\mathbf{D})$ functional calculi; moreover, $\mathscr{A}(T)$ and $\mathscr{A}\left(r(T-\alpha)^{-1}\right)$ are isometrically isomorphic with $H^{\infty}(\mathbf{D})$ (via their functional calculi; see [23]).

Define $\varphi(\lambda)=\sum_{n=0}^{\infty} a_{n} \lambda^{n}$ and $\psi(\lambda)=\sum_{n=1}^{\infty}\left(b_{-n} r^{-n}\right) \lambda^{n}$. By (4), $\varphi$ and $\psi$ are analytic on the open unit disk.

Claim. $\varphi, \psi \in H^{\infty}(\mathbf{D})$ and $A=\varphi(T)+\psi\left(r(T-\alpha)^{-1}\right)$.

Observe that, if this claim is true, then an analysis of the matrix of $\varphi(T)+$ $\psi\left(r(T-\alpha)^{-1}\right)$ indicates that $b_{n}=(1 / n !) \varphi^{(n)}(\alpha)$ for all $n=0,1,2, \ldots$ 
Write $b_{n}=(1 / n !) \varphi^{(n)}(\alpha)+\nu_{n} \quad\left(\nu_{n}\right.$ is "the error term"; $\left.n \geq 0\right)$. By induction, we obtain (use (5))

$$
\begin{aligned}
& A_{0,-1}=\alpha^{-1}\left[\varphi(\alpha)-a_{0}+\nu_{0}\right] T_{-1} \text {, } \\
& A_{1,-1}=\alpha^{-2}\left[\varphi(\alpha)-a_{0}-a_{1} \alpha+\nu_{0}\right] T_{0} T_{-1} \text {, } \\
& A_{n,-1}=\alpha^{-(n+1)}\left[\varphi(\alpha)-\sum_{i=0}^{n} a_{i} \alpha^{i}+\nu_{0}\right] T_{n-1} T_{n-2} \cdots T_{0} T_{-1},
\end{aligned}
$$

The coefficient is the sum of

$$
\alpha^{-(n+1)}\left[\varphi(\alpha)-\sum_{i=0}^{n} a_{i} \alpha^{i}\right]=\sum_{i=0}^{\infty} a_{i+n+1} \alpha^{i}
$$

(and the absolute values of these numbers are obviously uniformly bounded by some constant $C>0)$ and $\nu_{0} \alpha^{-(n+1)}$.

Thus, if $\nu_{0} \neq 0$, then we arrive at the contradiction

$$
\begin{aligned}
\|A\| & \geq \sup _{n}\left\|A_{n,-1}\right\| \geq \sup _{n}\left|\nu_{0} \alpha^{-(n+1)}\right| \cdot\left\|T_{n-1} T_{n-2} \cdots T_{0} T_{-1}\right\|-C \\
& \geq \sup _{n}\left|\nu_{0}[(1+|\alpha|) / 2]^{-(n+1)}\right|-C=\infty .
\end{aligned}
$$

Therefore $\nu_{0}=0$.

Another inductive argument shows that $\nu_{n}=0 \quad(n \geq 0)$; that is, $b_{n}$ is, indeed, equal to $(1 / n !) \varphi^{(n)}(\alpha)$ for all $n \geq 0$.

It follows that (use (4))

$$
\begin{aligned}
& \sum_{n=1}^{\infty}\left|b_{n}\right| \max _{j<-n}\left\|T_{j+n-1} T_{j+n-2} \cdots T_{j}\right\| \\
& \quad \leq \sum_{n=1}^{\infty}(1 / n !)\left|\varphi^{(n)}(\alpha)\right| \max _{j<-n}\left\|T_{j+n-1} T_{j+n-2} \cdots T_{j}\right\|<\infty
\end{aligned}
$$

because $|\alpha|+r<1, \max _{j<-n}\left\|T_{j+n-1} T_{j+n-2} \cdots T_{j}\right\|=O\left([r(1+\varepsilon)]^{n}\right)$ for all $\varepsilon>0$, and $\varphi$ has radius of convergence at least 1 .

This means that the operator

$$
A(1,1):=\left(A_{i j}\right)_{i, j<0}: \sum \bigoplus_{n<0} \mathscr{R}_{n} \rightarrow \sum \bigoplus_{n<0} \mathscr{R}_{n}
$$

can be written as the sum of two operators, $A(1,1)^{+}$and $A(1,1)^{-}$, where $A(1,1)^{+}\left(A(1,1)^{-}\right)$has the same entries as $\left(A_{i j}\right)_{i, j<0}$ for $i \leq j$ (for $i>j$, resp.) and the other entries equal to 0.

Another cumbersome calculation shows that $a_{-n}=(1 / n !) \psi^{(n)}\left(r(1-\alpha)^{-1}\right)$, so that

$$
\begin{aligned}
& \sum_{n=1}^{\infty}\left|a_{-n}\right| \max _{i \geq 0}\left\|\left(T_{i+n-1} T_{i+n-2} \cdots T_{i}\right)^{-1}\right\| \\
& \quad \leq \sum_{n=1}^{\infty}(1 / n !)\left|\psi^{(n)}\left(r(1-\alpha)^{-1}\right)\right| \max _{i \geq 0}\left\|\left(T_{i+n-1} T_{i+n-2} \cdots T_{i}\right)^{-1}\right\|<\infty
\end{aligned}
$$


because $\left|r(1-\alpha)^{-1}\right|<1, \max _{i \geq 0}\left\|\left(T_{i+n-1} T_{i+n-2} \cdots T_{i}\right)^{-1}\right\|=O\left((1+\varepsilon)^{n}\right)$ for all $\varepsilon>0$, and $\psi$ has radius of convergence at least 1 .

Thus, $A(0,0):=\left(A_{i j}\right)_{i, j \geq 0}$ can also be "split" along the main diagonal as $A(0,0)=A(0,0)^{+}+A(0,0)^{-}$, where $A(0,0)^{+}\left(A(0,0)^{-}\right)$has the same entries as $A(0,0)$ for $i \geq j$ (for $i<j$, resp.) and the other entries equal to 0 .

Let $A(1,0)=\left(A_{i j}\right)_{i \geq 0, j<0}$ and $A(0,1)=\left(A_{i j}\right)_{i<0, j \geq 0}$. We deduce from the above results that

$$
\begin{aligned}
A & =\left(\begin{array}{cc}
A(1,1) & A(1,0) \\
A(0,1) & A(0,0)
\end{array}\right)=\left(\begin{array}{cc}
A(1,1)^{+}+A(1,1)^{-} & A(1,0) \\
A(0,1) & A(0,0)^{+}+A(0,0)^{-}
\end{array}\right) \\
& =A^{+}+A^{-},
\end{aligned}
$$

where

$$
A^{+}=\left(\begin{array}{cc}
A(1,1)^{+} & 0 \\
A(0,1) & A(0,0)^{+}
\end{array}\right) \quad \text { and } \quad A^{-}=\left(\begin{array}{cc}
A(1,1)^{-} & A(1,0) \\
0 & A(0,0)^{-}
\end{array}\right) .
$$

It is obvious that both $A^{+}$and $A^{-}$commute with $T$. Since an element in the commutant of $T$ is uniquely determined by its 0 th column, a comparison of the corresponding matrices shows that $A^{+}=\varphi(T)$ and $A^{-}=\psi\left(r(T-\alpha)^{-1}\right)$, whence we deduce that $\varphi, \psi \in H^{\infty}(\mathbf{D})$ and $A=\varphi(T)+\psi\left(r(T-\alpha)^{-1}\right) \epsilon$ $\mathscr{A}^{a}(T)$.

Hence, $\mathscr{A}^{\prime}(T)=\mathscr{A}^{a}(T)$. (It is a straightforward exercise that every function $\omega$ in $H^{\infty}(\mathbf{D})$ admits a unique decomposition of the form $\omega(\lambda)=\varphi(\lambda)+$ $\psi\left(r(\lambda-\alpha)^{-1}\right)$, where $\varphi, \psi \in H^{\infty}(\mathbf{D})$ and $\left.\psi(0)=0.\right)$

The proof of Lemma 2.5 is now complete.

Now we are in a position to prove Proposition 2.1. The case $k=1$ is the contents of Lemma 2.5: $T=\left(\begin{array}{cc}r L+\alpha & 0 \\ C & R\end{array}\right)$ with respect to the decomposition $\mathscr{H}=\left(\sum \bigoplus_{n<0} \mathscr{R}_{n}\right) \oplus\left(\sum \bigoplus_{n \geq 0} \mathscr{R}_{n}\right)$, where $R$ is given by Lemma 2.3 and Remark 2.4(ii), $L$ is the adjoint of the similarly defined operator with the weight $T_{n}$ replaced by $T_{n}^{-1} \quad(n \geq 2)$ and

$$
C=\left(\begin{array}{ccc}
\cdots & 0 & r T_{-1} \\
& & 0 \\
& & \vdots
\end{array}\right)
$$

Assume that $k \geq 2$; then we define

$$
T=\left(\begin{array}{cccccc}
r_{k} L+\alpha_{k} & & & & & \\
& r_{k-1} L+\alpha_{k-1} & & & & \\
& & \ddots & & & \\
& & & r_{2} L+\alpha_{2} & & \\
& & & C_{2} L+\alpha_{1} & \\
C_{k} & C_{k-1} & \ldots & C_{1} & R
\end{array}\right)
$$

with respect to the decomposition $\mathscr{H}=\left\{\left(\sum \bigoplus_{n<0} \mathscr{R}_{n}\right) \oplus \cdots \oplus\left(\sum \bigoplus_{n<0} \mathscr{R}_{n}\right)\right.$ ( $k$ copies $)\} \oplus\left(\sum \bigoplus_{n \geq 0} \mathscr{R}_{n}\right)$ and $C_{j}$ is defined exactly as $C$, with $r$ replaced 
by $r_{j}, j=1,2, \ldots, k$. (Compare with the generalized Rota's model defined in [8].)

For each $h, 1 \leq h \leq k$,

$$
\sigma\left(\left(\begin{array}{cc}
r_{h} L+\alpha_{h} & 0 \\
C_{h} & R
\end{array}\right)\right)=\mathbf{D}^{-} \backslash \mathbf{D}\left(\alpha_{h}, r_{h}\right),
$$

which includes $\bigcup_{j \neq h} \sigma\left(r_{j} L+\alpha_{j}\right)=\bigcup_{j \neq h} \mathbf{D}\left(\alpha_{j}, r_{j}\right)^{-}$.

Since the latter set does not disconnect the plane, it is not difficult to prove that $\sigma(T)=\mathbf{D}^{-} \backslash \bigcup_{j=1}^{k} \mathbf{D}\left(\alpha_{j}, r_{j}\right)$; furthermore, $\sigma_{l e}(T)=\partial \sigma(T)$ and $\lambda-T$ is a semi-Fredholm operator with index equal to $-\operatorname{dim} \mathscr{R}$ and trivial kernel for all $\lambda \in \sigma(T) \backslash \sigma_{l e}(T)$.

Let $A=\left(B_{m n}\right)_{m, n=0}^{k}$ be the operator matrix of an element of $\mathscr{A}^{\prime}(T$ ) (for each pair $(m, n), B_{m n}$ can be written as an infinite operator matrix in the obvious way). We have $0=T A-A T=\left(M_{m n}\right)_{m, n=0}^{k}$, where

(1) $M_{j j}=\left[r_{j} L, B_{j j}\right]-B_{j 0} C_{j}, j=k, k-1, \ldots, 2,1$,

(2) $M_{00}=\left[R, B_{00}\right]+\sum_{j=1}^{k} C_{j} B_{j 0}$,

(3) $M_{i j}=\left(\alpha_{i}+r_{i} L\right) B_{i j}-B_{i j}\left(\alpha_{j}+r_{j} L\right)-B_{i 0} C_{j}, k \geq i, j \geq 1, i \neq j$,

(4) $M_{i 0}=\left(\alpha_{i}+r_{i} L\right) B_{i 0}-B_{i 0} R, k \geq i \geq 1$, and

(5) $M_{0 j}=\sum_{i=1}^{k} C_{i} B_{i j}+R B_{0 j}-B_{0 j}\left(\alpha_{j}+r_{j} L\right)-R C_{j}, k \geq j \geq 1$.

Exactly as in the proof of Lemma 2.3 and the first part of the proof of Lemma 2.5 , it can be shown that

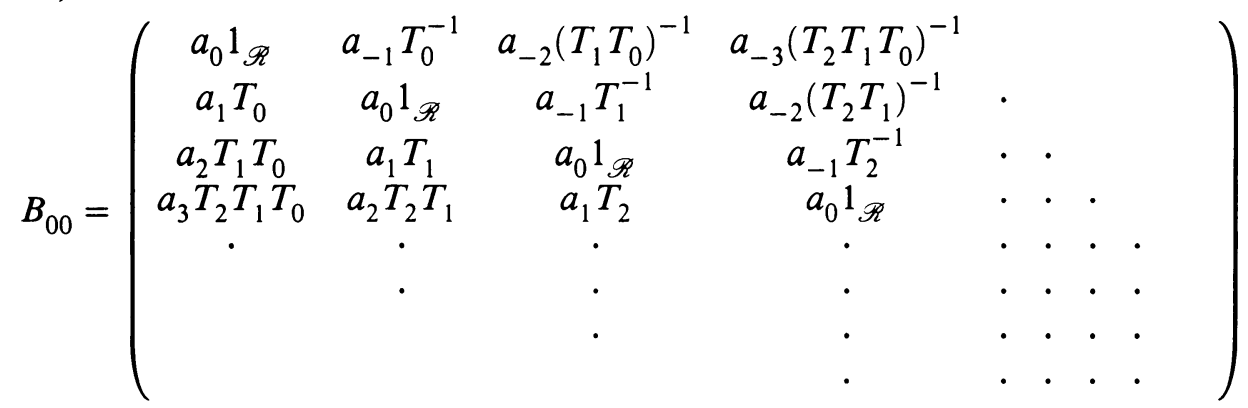

and

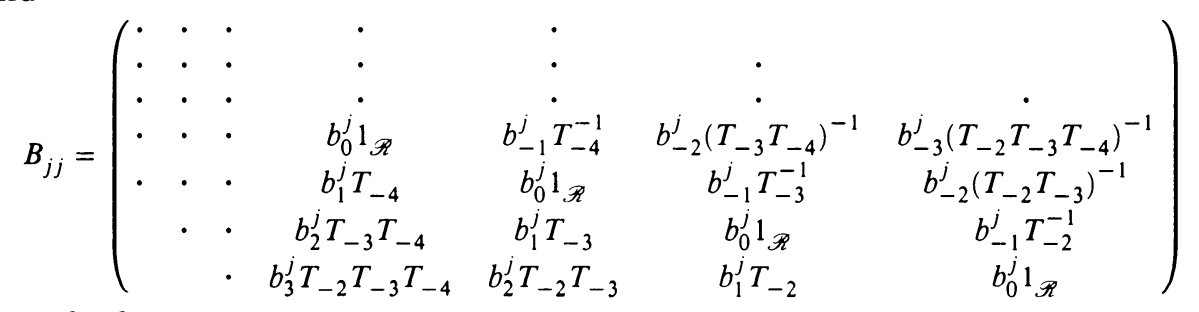

$(j=k, k-1, \ldots, 2,1$; use (1) and (2)).

Some simple estimates on the sizes of $a_{n} \quad(n \geq 0)$ and $b_{-n}^{j} \quad(n>0)$ indicate that, if we define

$$
\varphi(\lambda)=\sum_{n=0}^{\infty} a_{n} \lambda^{n} \quad \text { and } \quad \psi_{j}(\lambda)=\sum_{n=1}^{\infty}\left(b_{-n}^{j} r_{j}^{-n}\right) \lambda^{n},
$$


then $\varphi$ and $\psi_{j}(j=1,2, \ldots, k)$ are analytic on the open unit disk.

Furthermore, if in addition to (1) and (2) we consider also (3), (4), and (5), then we infer (as in the proof of Lemma 2.5) that the structure of $A$ is uniquely determined by the constants $\left\{a_{n}\right\}_{-\infty}^{\infty}$ and $\left\{b_{n}^{j}\right\}_{-\infty}^{+\infty}(j=1,2, \ldots, k)$; then, a double inductive argument shows that, indeed, this structure is uniquely determined only by $\left\{a_{n}\right\}_{n=0}^{\infty}$ and $\left\{b_{-n}^{j}\right\}_{n=1}^{\infty}(j=1,2, \ldots, k)$.

Thus (formally at least), we have $A=\varphi(T)+\sum_{j=1}^{k} \psi_{j}\left(r_{j}\left(T-\alpha_{j}\right)^{-1}\right)$.

The operator $T$ is contractive and the analysis of the matrix of $\left(T-\alpha_{j}\right)^{-1}$ indicates that $r_{j}\left(T-\alpha_{j}\right)^{-1}$ is also contractive; $(j=1,2, \ldots, k)$; furthermore, $T$ and $r_{j}\left(T-\alpha_{j}\right)^{-1}$ are actually $C_{00}$-contractions with (isometrically isomorphic) $H^{\infty}(\mathbf{D})$ functional calculi [23].

We conclude that $A$ is equal to the strong limit of

$$
A_{r}=\varphi(r T)+\sum_{j=1}^{k} \psi_{j}\left(r r_{j}\left(T-\alpha_{j}\right)^{-1}\right) \in \mathscr{A}^{a}(T), \quad \text { as } r \uparrow 1 .
$$

Hence, $\mathscr{A}^{\prime}(T)=\mathscr{A}^{a}(T)$.

The actual computations are a lot more cumbersome than the corresponding ones in Lemma 2.5, but the basic structure is the same: In $\varphi(T)$ there is a "main part" $\varphi(R)$, and

$$
\varphi\left(\alpha_{j}+r_{j} L\right)=\sum_{n=0}^{\infty} a_{n}\left(\alpha_{j}+r_{j} L\right)^{n}=\sum_{n=0}^{\infty}(1 / n !) \varphi^{(n)}\left(\alpha_{j}\right)\left(r_{j} L\right)^{n}
$$

is the "irrelevant part" (the series converges in the norm-topology, $j=1,2$, $\ldots, k)$. For each $h, 1 \leq h \leq k$, in $\psi_{h}\left(r_{h}\left(T-\alpha_{h}\right)^{-1}\right)$ the "main part" is

$$
\psi_{h}\left(r_{h} \text { “ }\left[\left(r_{h} L+\alpha_{h}\right)-\alpha_{h}\right]^{-1 ")}\right)=\psi_{h}\left(“ L^{-1 ")}\right.
$$

(the quotation marks mean that, although $L$ is not invertible, we can define $\psi_{h}\left(\right.$ “ $L^{-1}$ ”), where " $L^{-1}$ " is the unilateral operator weighted shift defined by

$$
\left(\begin{array}{cccccc}
\cdot \cdot & & & & \\
\cdot \cdot & & & & \\
& \cdot & \cdot & & & \\
& 0 & T_{4} & & \\
& & 0 & T_{3} & \\
& & & 0 & T_{2} \\
& & & & 0
\end{array}\right) \begin{gathered}
\cdot \\
\mathscr{R}_{-4} \\
\mathscr{R}_{-2} \\
\mathscr{R}_{-1}
\end{gathered}
$$

which "looks like the inverse" of the backward operator weighted shift $L)$. In this case the "irrelevant part" is the sum of $\psi_{h}\left(r_{h}{ }^{\prime \prime}\left(R-\alpha_{h}\right)^{-1}\right.$ ") and $\psi_{h}\left(r_{h}\left(r_{j} L-\alpha_{j}\right)^{-1}\right)$; these series also converge in the norm-topology and, moreover, $\psi_{h}\left(r_{h}\left(r_{j} L-\alpha_{j}\right)^{-1}\right)$ is actually a uniform limit of polynomials in $L$ for all $j \neq h$. Thus, the entries of the matrix of $B_{h h}$ above the main diagonal can 
only come from $\psi_{h}$ (" $L^{-1 ")}(h=1,2, \ldots, k)$. Similarly, the entries of the matrix $B_{00}$ on and below the main diagonal can only come from $\varphi(R)$.

The details of the constructions are left to the really dedicated reader.

The proof of Proposition 2.1 is now complete.

Remark 2.6. In Proposition 2.1, the operator $T$ is defined so that $T$ and $r_{j}\left(T-\alpha_{j}\right)^{-1}(j=1,2, \ldots, k)$ are $C_{00}$-contractions with $H^{\infty}(\mathbf{D})$ functional calculi in order to simplify the construction. But this is not strictly necessary. Instead of choosing $\left\{T_{n}\right\}_{n=0}^{\infty}$ as in Remark 2.4(ii), we can choose the sequence defined in Remark 2.4(i), and then justify that

$$
\begin{aligned}
\varphi(T) & =(\text { strong }) \lim _{r \uparrow 1} \sum_{n=0}^{\infty} a_{n}(r T)^{n} \\
& =(\text { strong }) \lim _{m \rightarrow \infty} \sum_{n=0}^{m}\left(1-\frac{n}{m+1}\right) a_{n} T^{n} \in \mathscr{A}(T)
\end{aligned}
$$

and

$$
\begin{aligned}
\psi_{j}\left(r_{j}\left(T-\alpha_{j}\right)^{-1}\right) & =(\text { strong }) \lim _{r \uparrow 1} \sum_{n=1}^{\infty} b_{-n}^{j}\left(T-\alpha_{j}\right)^{-n} \\
& =(\text { strong }) \lim _{m \rightarrow \infty} \sum_{n=0}^{m}\left(1-\frac{n}{m+1}\right) b_{-n}^{j}\left(T-\alpha_{j}\right)^{-n}
\end{aligned}
$$

$(j=1,2, \ldots, k)$ by using the same kinds of arguments as in [6] or [22].

In this case, $\mathscr{A}(T)$ is isometrically isomorphic with a certain Banach algebra $H^{\infty}(\mathbf{D} ; T)$ continuously embedded in $H^{\infty}(\mathbf{D})$ (norm-decreasing embedding). Since the family $\left\{T_{i+1} T_{i+2} \cdots T_{i+n}\right\}_{i, n=0}^{\infty}$ is not uniformly bounded, the embedding of $H^{\infty}(\mathrm{D} ; T)$ is strictly included in $H^{\infty}(\mathbf{D})$.

Proof of Proposition 2.2. Define $T$ as in the proof of Proposition 2.1, except that $\left\{T_{-n}\right\}_{n=2}^{\infty}$ is chosen as $\left\{T_{n}\right\}_{n=2}^{\infty}$ in Remark 2.4(iii). The entries of an operator $A$ commuting with $T$ are computed in exactly the same way as in the previous case, and these computations indicate that $b_{-n}^{j}=0$ for all $n \geq 1$ and all $j=1,2, \ldots, k$, because all the sequences $\left\{T_{-n}^{-1}\right\}_{n=2}^{\infty},\left\{T_{-n} T_{-(n+1)}\right\}_{n=2}^{\infty}$, $\left\{T_{-n} T_{-(n+1)} T_{-(n+2)}\right\}_{n=2}^{\infty}, \ldots$ are unbounded.

A fortiori, $a_{-n}=0$ for all $n \geq 1$, etc. Now it is easily seen that $A=\varphi(A) \in$ $\mathscr{A}(T)$, where $\varphi(\lambda)=\sum_{n=0}^{\infty} a_{n} \lambda^{n}$.

Hence, $\mathscr{A}^{\prime}(T)=\mathscr{A}(T)$.

Of course, the arguments of the proof of Propositions 2.1 and 2.2 can also be applied to the following "mixed" situation, as well:

Proposition 2.7. Let $\mathbf{D}$ denote the open unit disk, let $\left\{\mathbf{D}\left(\alpha_{j}, r_{j}\right)\right\}_{j=1}^{k}$ be a finite collection of open disks with pairwise disjoint closures such that $\mathbf{D}\left(\alpha_{j}, r_{j}\right)^{-} \subset$ D for all $j=1,2, \ldots, k$, and let $\left\{\mu_{1}, \mu_{2}, \ldots, \mu_{n}\right\}$ be a finite subset of $\mathbf{D} \backslash \bigcup_{j=1}^{k} \mathbf{D}\left(\alpha_{j}, r_{j}\right)^{-}$. 
Let $\Omega=\mathbf{D} \backslash\left[\left(\bigcup_{j=1}^{k} \mathbf{D}\left(\alpha_{j}, r_{j}\right)^{-}\right) \cup\left\{\mu_{1}, \mu_{2}, \ldots, \mu_{n}\right\}\right]$. Given an index $m$, $1 \leq m \leq \infty$, there exists $T$ in $\mathscr{L}(\mathscr{H})$ such that

(i) $\sigma(T)=\Omega^{-}$and $\sigma_{l e}(T)=\partial \Omega$;

(ii) $\operatorname{ker}(\lambda-T)=\{0\}$ and $\operatorname{dim} \operatorname{ker}(\lambda-T)^{*}=m$ for all $\lambda \in \Omega$;

(iii) $\lambda-T$ is a semi-Fredholm operator of index $-m$ for all $\lambda \in \Omega$, and

(iv) $\mathscr{A}^{a}(T)=\mathscr{A}^{\prime}(T)$ is isomorphic (but not necessarily isometric) with $H^{\infty}$ (interior $\left[\Omega^{-}\right]$).

\section{DENSITY OF OPERATORS WITH TINY COMMUTANTS}

Theorem 3.1. The class $\left\{T \in \mathscr{L}(\mathscr{H}): \mathscr{A}^{\prime}(T)=\mathscr{A}^{a}(T)\right\}$ is uniformly dense in $\mathscr{L}(\mathscr{H})$.

Proof. Recall that an analytic Cauchy domain is a nonempty, bounded, open subset of $\mathbf{C}$ whose boundary consists of finitely many pairwise disjoint regular analytic Jordan curves. An analytic Cauchy region is a connected analytic Cauchy domain.

Given $A$ in $\mathscr{L}(\mathscr{H})$ and $\varepsilon>0$, we can find $A_{1} \in \mathscr{L}(\mathscr{H})$ such that $\left\|A-A_{1}\right\|<\varepsilon, \sigma_{\text {lre }}\left(A_{1}\right)$ is the closure of an analytic Cauchy domain $\Omega$, and $\sigma\left(A_{1}\right)$ has only finitely many isolated points $\lambda_{1}, \lambda_{2}, \ldots, \lambda_{p}$ (see [2], [11], or [15]). Let $\Omega_{1}^{+}, \Omega_{2}^{+}, \ldots, \Omega_{m}^{+}\left(\Omega_{1}^{-}, \Omega_{2}^{-}, \ldots, \Omega_{n}^{-}\right)$be an enumeration of all those components of the semi-Fredholm domain $\rho_{s-F}\left(A_{1}\right)$ of $A_{1}$ such that ind $\left(\lambda-A_{1}\right)$ is positive (negative, resp.), and let $i_{h}\left(-i_{k}\right)$ be the index of $\lambda-A_{1}$ for $\lambda$ in $\Omega_{h}^{+}$(in $\Omega_{k}^{-}$, resp.).

Since $\rho_{s-F}\left(A_{1}\right)=\mathbf{C} \backslash \sigma_{l r e}\left(A_{1}\right)=\mathbf{C} \backslash \Omega^{-}$, and $\Omega$ is an analytic Cauchy domain, it readily follows that $\Omega_{1}^{+}, \Omega_{2}^{+}, \ldots, \Omega_{m}^{+}$and $\Omega_{1}^{-}, \Omega_{2}^{-}, \ldots, \Omega_{n}^{-}$are analytic Cauchy regions. According to [7, Theorem 2, p. 237] there exist "perforated disks" (as in Proposition 2.1) $\mathbf{D}_{1}^{+}, \mathbf{D}_{2}^{+}, \ldots, \mathbf{D}_{m}^{+}$and $\mathbf{D}_{1}^{-}, \mathbf{D}_{2}^{-}, \ldots, \mathbf{D}_{n}^{-}$and analytic functions $\varphi_{1}^{+}, \varphi_{2}^{+}, \ldots, \varphi_{m}^{+}$and $\varphi_{1}^{-}, \varphi_{2}^{-}, \ldots, \varphi_{n}^{-}$such that $\varphi_{h}^{+}$maps (a neighborhood of) the closure of $\left(\mathbf{D}_{h}^{+}\right)^{-}:=\left\{\lambda \in \mathbf{C}: \bar{\lambda} \in \mathbf{D}_{h}^{+}\right\}$conformally onto (a neighborhood of) the closure of $\left(\Omega_{h}^{+}\right)^{-}(h=1,2, \ldots, m)$ and $\varphi_{k}^{-}$maps (a neighborhood of) $\left(\mathbf{D}_{k}^{-}\right)^{-}$conformally onto (a neighborhood of) the closure of $\Omega_{k}^{-}(k=1,2, \ldots, n)$.

Let $R_{k}^{-}$be the operator constructed in Proposition 2.1 such that $\sigma\left(R_{k}^{-}\right)=$ $\left(\mathbf{D}_{k}^{-}\right)^{-}, \quad \sigma_{l e}\left(R_{k}^{-}\right)=\partial \mathbf{D}_{k}^{-}$, and $\operatorname{ind}\left(\lambda-R_{k}^{-}\right)=-i_{k}$ for all $\lambda \in \mathbf{D}_{k}^{-} \quad(k=$ $1,2, \ldots, m)$. Similarly, let $R_{h}^{+}=\left(R_{h}^{-}\right)^{*}$, where $R_{h}^{-}$is the operator given by Proposition 2.1 such that $\sigma\left(R_{h}^{-}\right)=\left(\mathbf{D}_{h}^{+}\right)^{-}, \sigma_{l e}\left(R_{h}^{-}\right)=\partial \mathbf{D}_{h}^{+}$, and $\operatorname{ind}\left(\lambda-R_{h}^{-}\right)=$ $-i_{h}$ for all $\lambda \in \mathbf{D}_{h}^{+}(h=1,2, \ldots, m)$.

The operators $\varphi_{h}^{+}\left(R_{h}^{+}\right)$and $\varphi_{k}^{-}\left(R_{k}^{-}\right)$are well-defined via Riesz-Dunford functional calculus, and it is straightforward to check that

$$
\sigma\left(\varphi_{h}^{+}\left(R_{h}^{+}\right)\right)=\left(\Omega_{h}^{+}\right)^{-}, \quad \sigma_{r e}\left(\varphi_{h}^{+}\left(R_{h}^{+}\right)\right)=\partial \Omega_{h}^{+},
$$




$$
\begin{aligned}
& \operatorname{ind}\left(\lambda-\varphi_{h}^{+}\left(R_{h}^{+}\right)\right)=i_{h} \text { and } \operatorname{ker}\left(\lambda-\varphi_{h}^{+}\left(R_{h}^{+}\right)\right)^{*}=\{0\} \quad \text { for all } \lambda \in \Omega_{h}^{+}, \\
& \mathscr{A}^{\prime}\left(\varphi_{h}^{+}\left(R_{h}^{+}\right)\right)=\mathscr{A}^{\prime}\left(R_{h}^{+}\right)=\mathscr{A}^{a}\left(R_{h}^{+}\right)=\mathscr{A}^{a}\left(\varphi_{h}^{+}\left(R_{h}^{+}\right)\right) \quad(h=1,2, \ldots, m),
\end{aligned}
$$

and

$$
\sigma\left(\varphi_{k}^{-}\left(R_{k}^{-}\right)\right)=\left(\Omega_{k}^{-}\right)^{-}, \quad \sigma_{l e}\left(\varphi_{k}^{-}\left(R_{k}^{-}\right)\right)=\partial \Omega_{k}^{-},
$$

$\operatorname{ind}\left(\lambda-\varphi_{k}^{-}\left(R_{k}^{-}\right)\right)=-i_{k}$ and $\operatorname{ker}\left(\lambda-\varphi_{k}^{-}\left(R_{k}^{-}\right)\right)=\{0\} \quad$ for all $\lambda \in \Omega_{k}^{-}$,

$$
\mathscr{A}^{\prime}\left(\varphi_{k}^{-}\left(R_{k}^{-}\right)\right)=\mathscr{A}^{\prime}\left(R_{k}^{-}\right)=\mathscr{A}^{a}\left(R_{k}^{-}\right)=\mathscr{A}^{a}\left(\varphi_{k}^{-}\left(R_{k}^{-}\right)\right) \quad(k=1,2, \ldots, n) \text {. }
$$

Clearly, the Riesz spectral invariant subspace $\mathscr{H}\left(A_{1} ; \lambda_{l}\right)$ (associated with the clopen subset $\left\{\lambda_{l}\right\}$ of $\left.\sigma\left(A_{1}\right)\right)$ is finite-dimensional: $1 \leq d_{l}:=\operatorname{dim} \mathscr{H}\left(A_{1} ; \lambda_{l}\right)<$ $\infty(l=1,2, \ldots, p)$. For each of the (finitely many) components $\Omega_{1}, \Omega_{2}, \ldots$, $\Omega_{t}$ of $\Omega$, let $\mathbf{D}\left(\beta_{s}, r_{s}\right)$ be a disk whose closure is contained in $\Omega_{s}(s=$ $1,2, \ldots, t)$.

Define

$$
\begin{aligned}
T= & \left\{\sum \bigoplus_{h=1}^{m} \varphi_{h}^{+}\left(R_{h}^{+}\right)\right\} \oplus\left\{\sum \bigoplus_{k=1}^{n} \varphi_{k}^{-}\left(R_{k}^{-}\right)\right\} \\
& \oplus\left\{\sum \bigoplus_{l=1}^{p}\left(\lambda_{l}+q_{d_{l}}\right)\right\} \oplus\left\{\sum \bigoplus_{s=1}^{t}\left(\beta_{s}+r_{s} S\right)\right\},
\end{aligned}
$$

where $q_{d}$ denotes the nilpotent Jordan cell of order $d$ and $S$ is the unilateral shift of multiplicity one. Since the sets $\sigma\left(\varphi_{h}^{+}\left(R_{h}^{+}\right)\right)=\left(\Omega_{h}^{+}\right)^{-}, \sigma\left(\varphi_{k}^{-}\left(R_{k}^{-}\right)\right)=$ $\left(\Omega_{k}^{-}\right)^{-}, \sigma\left(\lambda_{l}+q_{l}\right)=\left\{\lambda_{l}\right\}$, and $\sigma\left(\beta_{s}+r_{s} S\right)=\mathbf{D}\left(\beta_{s}, r_{s}\right)^{-}$are pairwise disjoint, it is not difficult to infer that

$$
\begin{aligned}
\mathscr{A}^{\prime}(T)= & \left\{\sum \bigoplus_{h=1}^{m} \mathscr{A}^{\prime}\left(R_{h}^{+}\right)\right\} \oplus\left\{\sum \bigoplus_{k=1}^{n} \mathscr{A}^{\prime}\left(R_{k}^{-}\right)\right\} \\
& \oplus\left\{\sum \bigoplus_{l=1}^{p} \mathscr{A}^{\prime}\left(q_{d_{l}}\right)\right\} \oplus\left\{\sum \bigoplus_{s=1}^{t} \mathscr{A}^{\prime}(S)\right\},
\end{aligned}
$$

which coincides with $\mathscr{A}^{a}(T)$.

By using the Similarity Orbit Theorem [2, Theorems 9.1 and 9.2], we see that $A_{1}$ can be uniformly approximated by operators similar to $T$.

Hence, there exists $A_{2}$ similar to $T$ such that $\left\|A-A_{2}\right\| \leq\left\|A-A_{1}\right\|+\| A_{1}-$ $A_{2} \|<2 \varepsilon$.

Clearly, $\mathscr{A}^{\prime}\left(A_{2}\right)=\mathscr{A}^{a}\left(A_{2}\right)$. Since $\varepsilon$ can be chosen arbitrarily small, we conclude that $A$ belongs to the closure of the set of operators with tiny commutants.

Hence, $\left\{T \in \mathscr{L}(\mathscr{H}): \mathscr{A}^{\prime}(T)=\mathscr{A}^{a}(T)\right\}$ is dense in $\mathscr{L}(\mathscr{H})$.

Remarks 3.2. (i) D. A. Herrero [8] and D. Voiculescu [24] have independently constructed extensions of the classical Rota's model for a linear operator [20]: given an analytic Cauchy $\Omega$, there exists an operator $M$ on a Hilbert space $\mathscr{K}$ such that for each $T \in \mathscr{L}(\mathscr{H})$ with $\sigma(T) \subset \Omega$ there is an invariant subspace $\mathscr{M}$ 
of $M$ so that

$$
M=\left(\begin{array}{cc}
M(T) & * \\
0 & T^{\prime}
\end{array}\right) \stackrel{\mathscr{M}}{\mathscr{K} \ominus \mathscr{M},}
$$

where $M(T)=M \mid \mathscr{M}$ is similar to $M$ and $T^{\prime}$ is similar to $T$.

The proofs given in [8], [24] (see also [11, Chapter 3]) indicate that $M$ can be chosen to be $\varphi(R)$, where $R$ is the operator given by Proposition 2.1 for a suitable perforated disk, with $\mathscr{R}$ an infinite-dimensional space, and $\phi$ is a conformal mapping from the closure of this perforated disk onto $\Omega^{-}$. This construction produces a model $M$ with the additional property that $\mathscr{A}^{\prime}(M)=$ $\mathscr{A}^{a}(M)$.

(ii) For $1 \leq \operatorname{dim} \mathscr{R} \leq \infty$, the above construction shows that for each Cauchy region $\Omega$ and each index $m(1 \leq m \leq \infty)$, there exists an operator $M=$ $M(\Omega,-m)$ such that $\sigma(M)=\Omega^{-}, \sigma_{l e}(M)=\partial \Omega$, ind $(\lambda-M)=-m$ and $\operatorname{ker}(\lambda-M)=\{0\}$ for all $\lambda \in \Omega$, and $\mathscr{A}^{\prime}(M)=\mathscr{A}^{a}(M)$ is isomorphic with $H^{\infty}(\Omega)$. In particular, $\mathscr{A}^{\prime}(M)$ does not contain any nontrivial idempotent. (By taking adjoints, we can construct analogous examples with $\operatorname{ind}(\lambda-M)=m$ and $\operatorname{ker}(\lambda-M)^{*}=\{0\}$ for all $\lambda \in \Omega$. If $m$ is finite, then $M$ is essentially normal, that is, $M^{*} M-M M^{*}$ is a compact operator.)

These kinds of operators play a very important role in the proof of the "approximate inverse of the Riesz decomposition theorem" given in [16]. Indeed, the constructions of [16] also include operators $T$ such that $T$ does not commute with any nontrivial idempotent, $\sigma(T)=\Omega^{-}, \sigma_{l e}(T)=\sigma_{r e}(T)=\partial \Omega$, $\operatorname{ind}(\lambda-T)=0$ and $\operatorname{dim} \operatorname{ker}(\lambda-T)=\operatorname{dim} \operatorname{ker}(\lambda-T)^{*}=1$ for all $\lambda \in \Omega$.

However (as we shall see later, in Corollary 5.4), given a Cauchy region $\Omega$, it is impossible to construct an operator $T$ such that $\sigma(T)=\Omega^{-}, \sigma_{l e}(T)=$ $\sigma_{r e}(T)=\partial \Omega, \operatorname{ind}(\lambda-T)=0$ and $\operatorname{dim} \operatorname{ker}(\lambda-T)=\operatorname{dim} \operatorname{ker}(\lambda-T)^{*}=1$ for all $\lambda \in \Omega$, and $\mathscr{A}^{\prime}(T)=\mathscr{A}^{a}(T)$ because such an operator commutes with "too many" rank-one operators.

\section{OPERATORS SATISFYING Lat $T=\operatorname{Lat} \mathscr{A}^{a}(T)$}

Theorem 4.1. Let $\rho_{s-F}^{ \pm}(T)=\left\{\lambda \in \rho_{s-F}(T): \operatorname{ind}(\lambda-T) \neq 0\right\}$. Then

$$
\begin{aligned}
\{T & \left.\in \mathscr{L}(\mathscr{H}): \mathscr{A}(T)=\mathscr{A}^{a}(T)\right\}^{-} \\
& =\left\{T \in \mathscr{L}(\mathscr{H}): \text { Lat } T=\text { Lat } \mathscr{A}^{a}(T)\right\}^{-} \\
& =\left\{A \in \mathscr{L}(\mathscr{H}): \text { if } \gamma(\text { Jordan curve }) \subset \rho_{s-F}^{ \pm}(A) \text {, then } \hat{\gamma} \subset \sigma(A)\right\} .
\end{aligned}
$$

(Here $\hat{\gamma}$ denotes the polynomial hull of $\gamma$, that is, the complement of the unbounded component of $\mathbf{C} \backslash \gamma$.)

Proof. Let $\mathscr{W}_{1}, \mathscr{W}_{2}$, and $\mathscr{W}_{3}$ denote the first, the second, and, respectively, the third subset of $\mathscr{L}(\mathscr{H})$ in the statement of the theorem. Since $\mathscr{A}(T)=\mathscr{A}^{a}(T)$ obviously imply Lat $T=\operatorname{Lat} \mathscr{A}^{a}(T)$, the inclusion $\mathscr{W}_{1} \subset \mathscr{W}_{2}$ is trivial.

The inclusion $\mathscr{W}_{2} \subset \mathscr{W}_{3}$ is an observation of $\mathrm{J}$. B. Conway (personal communication): Suppose Lat $T=\operatorname{Lat} \mathscr{A}^{a}(T)$ and $\gamma$ is a Jordan curve included in 
$\rho_{s-F}^{ \pm}(T)$. Obviously, ind $(\lambda-T)$ is independent of the particular $\lambda$ in the curve $\gamma$. Assume this index is negative.

Let $\mathscr{M}=\bigvee\left\{T^{k} y\right\}_{k=0}^{\infty}$ be the cyclic invariant subspace of $T$ generated by a nonzero vector $y \in \operatorname{ker}(\xi-T)^{*}$ (for some $\xi \in \gamma$ ); then $\lambda-T \mid \mathscr{M}$ is a Fredholm operator of index -1 for all $\lambda$ in $\gamma$. Since $\mathscr{M}$ is a cyclic subspace, it follows from [9], [12] (or [2, Chapter 11]) that $\lambda-T \mid \mathscr{M}$ is a Fredholm operator of index -1 for all $\lambda \in \hat{\gamma}$.

Therefore, $\hat{\gamma} \subset \sigma(T \mid \mathscr{M})$. But $\mathscr{M} \in$ Lat $T=$ Lat $\mathscr{A}^{a}(T)$, so that $\sigma(T \mid \mathscr{M}) \subset$ $\sigma(T)[17]$.

Hence, $\hat{\gamma} \subset \sigma(T)$.

By taking adjoints, we deduce that $\hat{\gamma} \subset \sigma(T)$ for every Jordan curve $\gamma \subset$ $\rho_{s-F}(T)$ such that $\operatorname{ind}(\lambda-T)>0$ for $\lambda \in \gamma$.

It only remains to show that $\mathscr{W}_{3} \subset \mathscr{W}_{1}$. Assume that $A \in \mathscr{W}_{3}$ and let $\varepsilon>0$ be given. First of all, we approximate $A$ by an operator $A_{1} \in \mathscr{W}_{3}$ $\left(\left\|A-A_{1}\right\|<\varepsilon\right)$ such that $\sigma_{\text {lre }}\left(A_{1}\right)$ is the closure of an analytic Cauchy domain $\Omega$, including $\sigma_{\text {lre }}(A), \sigma\left(A_{1}\right)=\sigma(A) \cup \Omega^{-}, \operatorname{ind}\left(\lambda-A_{1}\right)=\operatorname{ind}(\lambda-A)$ and $\operatorname{dim} \operatorname{ker}\left(\lambda-A_{1}\right)=\operatorname{dim} \operatorname{ker}(\lambda-A)$ for all nonisolated points $\lambda$ of $\rho_{s-F}\left(A_{1}\right) \cap$ $\sigma\left(A_{1}\right)$, and the restriction of $A_{1}$ to $\mathscr{H}\left(A_{1} ; \lambda\right)$ is similar to " $\lambda+$ nilpotent Jordan cell of order $\operatorname{dim} \mathscr{H}\left(A_{1} ; \lambda\right)$ " for each of the (finitely many) isolated points $\lambda$ of $\sigma\left(A_{1}\right)$ (see [11]).

Thus $A_{1}$ is similar to the direct sum of a cyclic operator $F$ acting on a finitedimensional space and an operator $B \in \mathscr{W}_{3}$ such that $\sigma(B)$ has no isolated points; moreover, $\sigma(F)$ is disjoint from $\left[\rho_{s-F}^{ \pm}(B)\right]^{\wedge}$.

Let $\Omega_{1}, \Omega_{2}, \ldots, \Omega_{m}$ be an enumeration of the components of $\Omega$, and let $\Phi_{1}, \Phi_{2}, \ldots, \Phi_{p}$ be an enumeration of the components of $\rho_{s-F}^{ \pm}(B)$. For each $i=1,2, \ldots, m$, let $N_{j}$ be any operator such that $\sigma_{l e}\left(N_{j}\right)=\sigma_{r e}\left(N_{j}\right)=\sigma\left(N_{j}\right)$ is a closed disk included in $\Omega_{j}$. For each $k=1,2, \ldots, p$, let $M_{k}$ be any operator such that $\sigma\left(M_{k}\right)=\left(\Phi_{k}\right)^{-}, \sigma_{l r e}\left(M_{k}\right)=\partial \Phi_{k}, \operatorname{ind}\left(\lambda-M_{k}\right)=\operatorname{ind}(\lambda-B)$ and $\min \left\{\operatorname{dim} \operatorname{ker}\left(\lambda-M_{k}\right), \operatorname{dim} \operatorname{ker}\left(\lambda-M_{k}\right)^{*}\right\}=0$ for all $\lambda \in \Phi_{k}$. (These operators can be constructed, e.g., as in the proof of Theorem 3.1, or by using the results of [11, Chapter 3].)

Finally, if $\Psi_{1}, \Psi_{2}, \ldots, \Psi_{t}$ is an enumeration of the bounded components of $\mathbf{C} \backslash\left[\bigcup_{k=1}^{p} \Phi_{k}\right]^{-}$, then for each $h=1,2, \ldots, t$ we choose an operator $L_{h}$ such that $\sigma\left(L_{h}\right)=\left(\Psi_{h}\right)^{-}, \sigma_{l e}\left(L_{h}\right)=\sigma_{r e}\left(L_{h}\right)=\partial \Psi_{h}$, and $\operatorname{ind}\left(\lambda-L_{h}\right)=0$ and $\operatorname{dim} \operatorname{ker}\left(\lambda-L_{h}\right)=\operatorname{dim} \operatorname{ker}\left(\lambda-L_{h}\right)^{*}=1$ for all $\lambda \in \Psi_{h}$.

Let

$$
R=F \oplus\left\{\sum \bigoplus_{j=1}^{m} N_{h}\right\} \oplus\left\{\sum \bigoplus_{k=1}^{p} M_{k}\right\} \oplus\left\{\sum \bigoplus_{h=1}^{t} L_{h}\right\} .
$$

By construction, $\sigma(R)=\sigma(F) \cup\left\{\left(\bigcup_{j=1}^{m} \sigma\left(N_{j}\right)\right) \cup\left(\bigcup_{k=1}^{p} \Phi_{k}\right) \cup\left(\bigcup_{h=1}^{t} \Psi_{h}\right)\right\}^{-}$does not disconnect the plane; therefore (by using Runge's theorem [5]), $\mathscr{A}^{a}(R)=$ $\mathscr{A}(R)$. 
Moreover, $\sigma(R)$ is a subset of $\sigma\left(A_{1}\right), \rho_{s-F}(R) \supset \rho_{s-F}\left(A_{1}\right)$, and $\operatorname{ind}(\lambda-R)=\operatorname{ind}\left(\lambda-A_{1}\right)$ and $\min \left\{\operatorname{dim} \operatorname{ker}(\lambda-R)^{k}, \operatorname{dim} \operatorname{ker}\left[(\lambda-R)^{*}\right]^{k}\right\} \leq$ $\min \left\{\operatorname{dim} \operatorname{ker}\left(\lambda-A_{1}\right)^{k}, \operatorname{dim} \operatorname{ker}\left[\left(\lambda-A_{1}\right)^{*}\right]^{k}\right\}$ for all $\lambda \in \rho_{s-F}\left(A_{1}\right)$ and all $k \geq 1$ (to see this, use that $\left.A_{1} \in \mathscr{W}_{3} !\right)$, every component of $\sigma_{l e}\left(A_{1}\right) \cap \sigma_{r e}\left(A_{1}\right)$ meets the perfect set $\sigma_{l e}(R) \cap \sigma_{r e}(R), \sigma\left(A_{1}\right)$ and $\sigma(R)$ have exactly the same isolated points, and $\operatorname{dim} \mathscr{H}\left(A_{1} ; \lambda\right)=\operatorname{dim} \mathscr{H}(R ; \lambda)$ for each such point.

By the Similarity Orbit Theorem [2, Theorems 9.1 and 9.2], there exists $A_{2}$ similar to $R$ such that $\left\|A-A_{2}\right\| \leq\left\|A-A_{1}\right\|+\left\|A_{1}-A_{2}\right\|<2 \varepsilon$.

Since $\mathscr{A}^{a}(R)=\mathscr{A}(R)$ and $\varepsilon$ can be chosen arbitrarily small, we conclude that $A \in \mathscr{W}_{1}$.

The proof of Theorem 4.1 is now complete.

Remarks 4.2. (i) An easy corollary of the results of [3] (see also [1 and 11, Chapter 4]) is that for each $R$ in $\mathscr{L}(\mathscr{H})$ there is a compact operator $K$ such that $\sigma(R-K)$ does not disconnect the plane, and therefore $\mathscr{A}^{a}(R-K)=$ $\mathscr{A}(R-K)$. Thus, if $\mathscr{K}(\mathscr{H})$ denotes the ideal of all compact operators, then $\left\{T \in \mathscr{L}(\mathscr{H}): \mathscr{A}^{a}(T)=\mathscr{A}(T)\right\}+\mathscr{K}(\mathscr{H})=\mathscr{L}(\mathscr{H})$.

(That is, $\left\{T \in \mathscr{L}(\mathscr{H}): \mathscr{A}^{a}(T)=\mathscr{A}(T)\right\}$ "essentially" coincides with $\mathscr{L}(\mathscr{H})$.)

(ii) Given $R$ in $\mathscr{L}(\mathscr{H})$, we have

$$
\begin{aligned}
& \operatorname{dist}\left[R,\left\{T: \mathscr{A}^{a}(T)=\mathscr{A}(T)\right\}\right] \\
& \quad \inf \{\|B\|: B \in \mathscr{L}(\mathscr{H}) \text { and } \sigma(R-B) \text { does not disconnect the plane }\} \\
& \leq \inf \left\{\|K\|: K \in \mathscr{K}(\mathscr{H}) \text { and for each Jordan curve } \gamma \subset \rho_{s-F}(R)\right. \\
& \text { such that } \operatorname{ind}(\lambda-R) \neq 0 \text { for } \lambda \text { in } \gamma, \hat{\gamma} \subset \sigma(R-K)\} .
\end{aligned}
$$

Both the equality and the inequality can be justified by using the proof of Theorem 4.1. (The details are left to the reader.)

The author conjectures that the inequality is actually an equality. An upper estimate for the second infimum can be easily derived from the results of [13].

(iii) By using the theorem on density of operators with "bad properties" [11, Theorem 3.51], it is not difficult to see that all the classes $\{T \in \mathscr{L}(\mathscr{H}): \mathscr{A}(T) \neq$ $\left.\mathscr{A}^{a}(T)\right\}$ and $\left\{T \in \mathscr{L}(\mathscr{H})\right.$ : Lat $T \neq$ Lat $\left.\mathscr{A}^{a}(T)\right\}$ (and the classes similarly defined with $\mathscr{A}(T)$ and $\mathscr{A}^{a}(T)$ replaced by any other pair of algebras) are dense in $\mathscr{L}(\mathscr{H})$.

Furthermore, if $B$ is any operator such that $\sigma(B)=\{\lambda \in \mathbf{C}: 1 \leq|\lambda| \leq 2\}$ and $\lambda-B$ is a Fredholm operator of index 1 for all $\lambda$ with $1<|\lambda|<2$, then $\mathscr{A}\left(B^{\prime}\right) \neq \mathscr{A}^{a}\left(B^{\prime}\right)$ for all $B^{\prime}$ close enough to $B$. By using this observation and the proof of [11, Theorem 3.51], we deduce that $\{T \in \mathscr{L}(\mathscr{H}): \mathscr{A}(T)=$ $\left.\mathscr{A}^{a}(T)\right\}^{-}$is nowhere dense in $\mathscr{L}(\mathscr{H})$.

A fortiori, so is $\{T \in \mathscr{L}(\mathscr{H}) \text { : Lat } T=\text { Hyperlat } T\}^{-}$(and the closure of any other class between these two). 


\section{The CASE Lat $T=$ Hyperlat $T$}

Proposition 5.1. $\left\{A \in \mathscr{L}(\mathscr{H})\right.$ : if $\gamma$ (Jordan curve) $\subset \rho_{s-F}^{ \pm}(A)$, then $\operatorname{ind}(\lambda-A)$ is constant for $\left.\lambda \in \hat{\gamma} \cap \rho_{s-F}(A)\right\}$

$$
\begin{aligned}
& \subset\left\{T \in \mathscr{L}(\mathscr{H}): \mathscr{A}(T)=\mathscr{A}^{\prime}(T)\right\}^{-} \\
& \subset\{T \in \mathscr{L}(\mathscr{H}): \text { Lat } T=\text { Hyperlat } T\}^{-} \\
& \subset\left\{A \in \mathscr{L}(\mathscr{H}): \text { if } \gamma(\text { Jordan curve }) \subset \rho_{s-F}^{ \pm}(A),\right. \\
& \left.\quad \text { then } \hat{\gamma} \cap \rho_{s-F}(A) \subset \rho_{s-F}^{ \pm}(A)\right\} \\
& \subset\left\{T \in \mathscr{L}(\mathscr{H}): \mathscr{A}(T)=\mathscr{A}^{a}(T)\right\}^{-} .
\end{aligned}
$$

Furthermore, the first and the last inclusions are proper.

We shall need an auxiliary result which has some interest in itself. Recall that $\mu \in \rho_{s-F}(T)$ is a singular point of the semi-Fredholm domain (in the sense of $\mathrm{C}$. Apostol [1]) if the mapping $\lambda \rightarrow P_{\operatorname{ker}(\lambda-T)}$ (= orthogonal projection of $\mathscr{H}$ onto $\operatorname{ker}(\lambda-T))$ is discontinuous at $\lambda=\mu$. In this case, $T=W[(\mu+Q) \oplus B] W^{-1}$, where $W$ is invertible, $Q$ is a Jordan nilpotent (acting on a finite-dimensional space $\mathscr{R}$ ), and $\mu$ is not singular for $B$ (see [1], [18], or [11, Chapter 3]).

Proposition 5.2. (i) If $T \in \mathscr{L}(\mathscr{H})$ and Lat $\mathscr{A}^{\prime \prime}(T)=$ Hyperlat $T$, then $\lambda \rightarrow$ $P_{\operatorname{ker}(\lambda-T)}$ is continuous at $\lambda=\mu$ for all interior points $\mu$ of $\sigma(T) \cap \rho_{s-F}(T)$; that is, the only singularities of the semi-Fredholm domain of $T$ are the isolated points of $\sigma(T) \backslash \sigma_{e}(T)$.

Furthermore, if $\lambda \in \sigma(T) \backslash \sigma_{e}(T)$ is isolated, then $T \mid \mathscr{H}(T ; \lambda)$ is a cyclic operator (on the finite-dimensional space $\mathscr{H}(T ; \lambda))$.

(ii) If Lat $\mathscr{A}^{a}(T)=$ Hyperlat $T$, then $\min \left\{\operatorname{dim} \operatorname{ker}(\lambda-T), \operatorname{dim} \operatorname{ker}(\lambda-T)^{*}\right\}=$ 0 for each interior point $\lambda$ of $\sigma(T) \cap \rho_{s-F}(T)$. In particular, $\mu-T$ is invertible for each interior point $\mu$ of $\sigma(T) \cap\left\{\lambda \in \rho_{s-F}(T): \operatorname{ind}(\lambda-T)=0\right\}$.

Proof. (i) If $\mu$ is a singular point of $\rho_{s-F}(T)$ and $\mu$ belongs to the interior of $\sigma(T)$, then $T$ has the above described form and $\lambda-B$ is a semi-Fredholm operator with nontrivial kernel (or nontrivial cokernel) for all $\lambda$ in some neighborhood of $\mu$. To simplify the notation, we can directly assume that $W=1$ and $\mu=0$, that is, $T=Q \oplus B$.

It is obvious that $T$ commutes with the orthogonal projection $P_{\mathscr{R}}$ (of $\mathscr{H}$ onto $\mathscr{R})$, and therefore $\mathscr{R}, \mathscr{H} \ominus \mathscr{R} \in$ Lat $\mathscr{A}^{\prime \prime}(T)$. If $f$ is a unit vector in $\operatorname{ker} Q^{*}$ and $g$ is a unit vector in $\operatorname{ker} B$, and we define the rank-one operator $g \otimes f^{*} \in \mathscr{L}(\mathscr{H})$ by $g \otimes f^{*}(x)=\langle x, f\rangle g$, then

$$
T\left(g \otimes f^{*}\right)=(B g) \otimes f^{*}=0 \quad \text { and }\left(g \otimes f^{*}\right) T=g \otimes\left(Q^{*} f\right)^{*}=0,
$$

so that $g \otimes f^{*}$ commutes with $T$. But $g \otimes f^{*}$ does not commute with $P_{\mathscr{R}}$, and therefore $g \otimes f^{*} \notin \mathscr{A}^{\prime \prime}(T)$.

Observe that $g=g \otimes f^{*}(f) \in g \otimes f^{*}(\mathscr{R})$, but $g \perp \mathscr{R}$.

Therefore, $\mathscr{R} \in$ Lat $\mathscr{A}^{\prime \prime}(T)$, but $\mathscr{R} \notin$ Hyperlat $T$. 
(If $\operatorname{ker} B=\{0\}$, then we apply the same argument to $T^{*}$, instead of $T$.)

An operator $Q$ acting on a finite-dimensional space satisfies $\mathscr{A}^{\prime \prime}(Q)=\mathscr{A}^{\prime}(Q)$ if and only if $Q$ is cyclic, whence we obtain the second statement (see, e.g., [11, Chapter 2]).

(ii) Now assume that $T$ is semi-Fredholm and 0 is an interior point of $\sigma(T)$. If 0 is singular, then Lat $\mathscr{A}^{a}(T) \neq$ Hyperlat $T$, by (i).

If 0 is not singular and $\operatorname{ker}(\lambda-T) \neq\{0\} \neq \operatorname{ker}(\lambda-T)^{*}$ for all $\lambda$ in the component $\Omega$ of $\rho_{s-F}(T)$ containing the origin; then we pick unit vectors $g \in$ $\operatorname{ker} T$ and $f \in \operatorname{ker} T^{*}$. We can directly assume that $\operatorname{ker} T$ is finite-dimensional. (If not, replace $T$ by $T^{*}$.) This guarantees that for each invariant subspace $\mathscr{M},(T \mid \mathscr{M}) \mid\left[\operatorname{ker}(T \mid \mathscr{M})^{\perp}\right]$ is bounded below.

Let $\mathscr{M}=\left\{A f: A \in \mathscr{A}^{a}(T)\right\}^{-} \in \operatorname{Lat} \mathscr{A}^{a}(T)$. Since $\mathscr{M}$ is a rationally cyclic invariant subspace, $T$ is semi-Fredholm with ind $T>\infty$, and $f \in \operatorname{ker} T^{*}=$ $(\operatorname{ran} T)^{\perp}$, it follows from [9], [12], or [2, Chapter 11] that $\lambda-T \mid \mathscr{M}$ is a Fredholm operator with trivial kernel and index equal to -1 for all $\lambda \in \Omega$. This means, in particular, that $g \notin \mathscr{M}$.

But $g \otimes f^{*} \in \mathscr{A}^{\prime}(T)$ and $g=g \otimes f^{*}(f) \in g \otimes f^{*}(\mathscr{M})$.

Therefore, $\mathscr{M} \in$ Lat $\mathscr{A}^{a}(T)$, but $\mathscr{M} \notin$ Hyperlat $T$.

Now the result follows by applying the above argument to $\mu-T$ for each interior point $\mu$ of $\rho_{s-F}(T) \cap \sigma(T)$.

Let $f$ and $g$ be as in the second part of the proof. It is easy to construct examples where $\mathscr{N}=\left\{B f: B \in \mathscr{A}^{\prime \prime}(T)\right\}^{-}\left(\in\right.$ Lat $\left.\mathscr{A}^{\prime \prime}(T)\right)$ satisfies that $\operatorname{ind}(T \mid \mathcal{N})=-m$ for some $m, 2 \leq m \leq \infty$ (see, e.g., [10]). Thus, $\mathscr{M}$ is a proper subspace of $\mathscr{N}$, in general.

Question 5.3. Let $f, g, \mathscr{M}$, and $\mathscr{N}$ be as above. Does $g \otimes f^{*}$ always belong to $\mathscr{A}^{\prime}(T) \backslash \mathscr{A}^{\prime \prime}(T)$ ? Does $\mathscr{N} \in$ Lat $\mathscr{A}^{\prime \prime}(T) \backslash$ Hyperlat $T$ ?

Corollary 5.4. If $\operatorname{ker}(\lambda-T)$ and $\operatorname{ker}(\lambda-T)^{*}$ are nontrivial for all $\lambda$ in some open disk included in $\rho_{s-F}(T)$, then $\mathscr{A}^{a}(T) \neq \mathscr{A}^{\prime}(T)$ and Lat $\mathscr{A}^{a}(T) \neq$ Hyperlat $T$.

Now we are in a position to prove Proposition 5.1. The second and the fourth inclusion are obvious, and Theorem 4.1 indicates that the fourth inclusion is actually proper (for instance, if $\sigma(A)=\mathbf{D}^{-}, \sigma_{e}(A)=\{\lambda:|\lambda|=1$ or $|\lambda|=1 / 2\}$, $\operatorname{ind}(\lambda-A)=0$ for $|\lambda|<1 / 2$, and $\operatorname{ind}(\lambda-A)=1$ for $1 / 2<|\lambda|<1$, then $\mathscr{A}(A)=\mathscr{A}^{a}(A)$, and $\gamma=\{\lambda \in \mathbf{C}:|\lambda|=3 / 2\} \subset \rho_{s-F}^{ \pm}(A)$, but $\hat{\gamma} \cap \rho_{s-F}(A) \not \subset$ $\rho_{s-F}^{ \pm}(A)$.

The third inclusion follows by Corollary 5.4. If $\gamma$ (Jordan curve) $\subset \rho_{s-F}^{ \pm}(A)$ and $\operatorname{ind}(\lambda-A)=0$ for some $\lambda \in \hat{\gamma} \cap \rho_{s-F}(A)$, then every $T$ close enough to $A$ has the same properties [4], [11, Chapter 1]. If $\lambda-T$ (or $\lambda^{\prime}-T$ for some $\lambda^{\prime}$ close enough to $\lambda$ ) is invertible, then Lat $T \neq \operatorname{Lat} \mathscr{A}^{a}(T)$, by Theorem 4.1. If $\operatorname{ker}(\mu-T) \neq\{0\} \neq \operatorname{ker}(\mu-T)^{*}$ for all $\mu$ on some neighborhood of $\lambda$, then Lat $\mathscr{A}^{a}(T) \neq$ Hyperlat $T$, by Corollary 5.4. Thus, $A$ cannot be approximated by operators satisfying Lat $T=$ Hyperlat $T$. 
Let $\mathscr{W}$ denote the first of the five sets described in the statement of the proposition. Let $A \in \mathscr{W}$ and let $\varepsilon>0$ be given. First of all, we find $A_{1} \in \mathscr{W}$, with $\left\|A_{1}-A\right\|<\varepsilon$, such that $\sigma_{l r e}\left(A_{1}\right)$ is the closure of an analytic Cauchy domain $\Omega$, and $\sigma\left(A_{1}\right)$ has only finitely many isolated points (see [11]).

Since $A_{1} \in \mathscr{W}$, we can find a finite collection $\Phi_{1}, \Phi_{2}, \ldots, \Phi_{m}$ of simply connected analytic Cauchy regions, with pairwise disjoint closures, such that $\partial \Phi_{j} \subset \Omega$ and $\operatorname{ind}\left(\lambda-A_{1}\right) \equiv m_{j} \neq 0$ for $\lambda \in \Phi_{j} \cap \rho_{s-F}\left(A_{1}\right)$, and $\operatorname{ind}\left(\lambda-A_{1}\right)=0$ for all $\lambda \in \rho_{s-F}\left(A_{1}\right) \backslash \bigcup_{j=1}^{m} \Phi_{j}$.

Let $\Omega_{1}, \Omega_{2}, \ldots, \Omega_{n}$ be an enumeration of the components of $\Omega$. If $\Omega_{k} \subset$ $\Phi_{j}$ (for some $j$ ), then we choose a closed disk $\Delta_{k} \subset \Omega_{k}$. If $\Omega_{k}$ is not included in the union of the $\Phi_{j}$ 's, then we choose a closed disk $\Delta_{k} \subset \Omega_{k} \backslash\left(\bigcup_{j=1}^{m} \Phi_{j}\right)^{-}$. Without loss of generality, we can assume that the $\Omega_{k}$ 's are ordered so that $\Omega_{k} \subset \bigcup_{j=1}^{m} \Phi_{j}$ if and only if $1 \leq k \leq p \quad(\leq n)$.

Let $N_{k}$ be a normal operator such that $\sigma\left(N_{k}\right)=\Delta_{k}(k=1,2, \ldots, n)$.

By construction, $A_{1}$ is similar to the direct sum of an operator $F$ acting on a finite-dimensional space $(\sigma(F)$ is the set of isolated points of $\sigma(A))$, and an operator $B$ such that $\sigma(B)=\sigma(A) \backslash \sigma(F)$. Without loss of generality, we can directly assume that $F$ is cyclic, and therefore $\mathscr{A}(F)=\mathscr{A}^{\prime}(F)$.

Let $L \in \mathscr{L}(\mathscr{H})$ be any operator of the form

$$
L=F \oplus\left\{\sum \bigoplus_{k=1}^{n} N_{k}\right\} \oplus\left\{\sum \bigoplus_{j=1}^{m} L_{j}\right\},
$$

where $\sigma\left(L_{j}\right)=\left(\Phi_{j}\right)^{-}, \sigma_{\text {lre }}\left(L_{j}\right)=\partial \Phi_{j}$, and $\operatorname{ind}\left(\lambda-L_{j}\right)=m_{j}$ and

$$
\min \left\{\operatorname{dim} \operatorname{ker}\left(\lambda-L_{j}\right), \operatorname{dim} \operatorname{ker}\left(\lambda-L_{j}\right)^{*}\right\}=0
$$

for all $\lambda \in \Phi_{j}, j=1,2, \ldots, m$.

The Similarity Orbit Theorem implies that there exists $A_{2}$ similar to $L$ such that $\left\|A_{1}-A_{2}\right\|<\varepsilon$.

For each $t \geq 1$, let $\left\{\mu_{r}^{t}\right\}$ be a set of $p t$ distinct points such that exactly $t$ of these points lie in $\Delta_{k}(k=1,2, \ldots, p)$.

For each $j=1,2, \ldots, m$, let $\phi_{j}$ be a conformal mapping from $\mathbf{D}^{-}$onto $\left(\Phi_{j}\right)^{-}$, and let $\Delta_{j, t}=\phi_{j}^{-1}\left(\Phi_{j} \cap\left\{\mu_{r}^{t}\right\}\right)$.

By Proposition 2.2, for each $j$ we can construct $R_{j, t}$ such that $\sigma\left(R_{j, t}\right)=$ $\mathbf{D}^{-}, \sigma_{l r e}\left(R_{j, t}\right)=\partial \mathbf{D} \cup \Delta_{j, t}$, and $\operatorname{ind}\left(\lambda-R_{j, t}\right)=m_{j}$ and

$$
\min \left\{\operatorname{dim} \operatorname{ker}\left(\lambda-R_{j, t}\right), \operatorname{dim} \operatorname{ker}\left(\lambda-R_{j, t}\right)^{*}\right\}=0
$$

for all $\lambda \in \mathbf{D} \backslash \Delta_{j, t}$. (This is obvious for $m_{j}<0$; if $m_{j}>0$, then we construct $R_{j, t}$ by taking suitable adjoints.)

Let

$$
T_{t}=F \oplus\left\{\sum \bigoplus_{k=p+1}^{n} N_{k}\right\} \oplus\left\{\sum \bigoplus_{j=1}^{n} \phi_{j}\left(R_{j, t}\right)\right\} .
$$


It is immediate that $\sigma\left(T_{t}\right)=\sigma(F) \cup\left(\bigcup_{k=p+1}^{n} \Delta_{k}\right) \cup\left(\bigcup_{j=1}^{m} \Phi_{j}\right)^{-}$, does not disconnect the plane,

$$
\begin{aligned}
\sigma_{l r e}\left(T_{t}\right) & =\left(\bigcup_{k=p+1}^{n} \Delta_{k}\right) \cup\left\{\bigcup_{j=1}^{m}\left[\partial \Phi_{j} \cup\left(\Phi_{j} \cap\left\{\mu_{r}^{t}\right\}\right)\right]\right\} \\
& \subseteq\left(\bigcup_{k=1}^{n} \Delta_{k}\right) \cup\left(\bigcup_{j=1}^{m} \partial \Phi_{j}\right) \subset \Omega,
\end{aligned}
$$

and $\operatorname{ind}\left(\lambda-T_{t}\right)=m_{j}$ and $\min \left\{\operatorname{dim} \operatorname{ker}\left(\lambda-T_{t}\right), \operatorname{dim} \operatorname{ker}\left(\lambda-T_{t}\right)^{*}\right\}=0$ for all $\lambda \in \Phi_{j} \backslash\left\{\mu_{r}^{t}\right\}$; moreover,

$$
\mathscr{A}^{\prime}\left(T_{t}\right)=\mathscr{A}^{\prime}(F) \oplus\left\{\sum \bigoplus_{k=p+1}^{n} \mathscr{A}^{\prime}\left(N_{k}\right)\right\} \oplus\left\{\sum \bigoplus_{j=1}^{n} \mathscr{A}^{\prime}\left(R_{j, t}\right)\right\}=\mathscr{A}\left(T_{t}\right) .
$$

The Similarity Orbit Theorem indicates that if $M_{t}$ is a diagonal normal operator of uniform infinite multiplicity such that $\sigma\left(M_{t}\right)=\sigma_{e}\left(M_{t}\right)=\left\{\mu_{r}^{t}\right\}$, then

$$
L_{t}=F \oplus\left\{\sum \bigoplus_{k=p+1}^{n} N_{k}\right\} \oplus M_{t} \oplus\left\{\sum \bigoplus_{j=1}^{m} L_{j}\right\}
$$

can be uniformly approximated by operators similar to $T_{t}$. Furthermore, for $t$ large, we can also approximate $\sum \bigoplus_{k=1}^{p} N_{k}$ by operators similar to $M_{t}$ (see [11, Chapter 5]).

Thus, if $t$ is sufficiently large, then we can find $A_{3}$ similar to $T_{t}$ such that $\left\|A_{2}-A_{3}\right\|<\varepsilon$.

Since $\left\|A-A_{3}\right\|<3 \varepsilon, \mathscr{A}^{\prime}\left(A_{3}\right)=\mathscr{A}\left(A_{3}\right)$, and $\varepsilon$ can be chosen arbitrarily small, we conclude that $A$ is the uniform limit of a sequence of operators $\left\{A_{t}\right\}_{t=1}^{\infty}$ satisfying the condition $\mathscr{A}^{\prime}\left(A_{t}\right)=\mathscr{A}\left(A_{t}\right)$ for all $t=1,2, \ldots$.

Hence, $\mathscr{W} \subset\left\{T \in \mathscr{L}(\mathscr{H}): \mathscr{A}(T)=\mathscr{A}^{\prime}(T)\right\}^{-}$.

It only remains to show that the first inclusion is also proper. This follows immediately from the following.

Example 5.5. Let $B$ denote the bilateral weighted shift defined by

$$
B e_{n}=\left\{\begin{array}{l}
e_{n+1} \quad(n \geq 0), \\
(1 /|n|) e_{n+1} \quad(n<0),
\end{array}\right.
$$

with respect to the orthonormal basis $\left\{e_{n}\right\}_{-\infty}^{+\infty}$ of $\mathscr{H}$, and let $\mathscr{H}_{+}=\bigvee\left\{e_{n}\right\}_{n \geq 0}$ and $\mathscr{H}_{-}=\bigvee\left\{e_{n}\right\}_{n<0}$.

Define $T \in \mathscr{L}(\mathscr{H} \oplus \mathscr{H})$ by

$$
T=\left(\begin{array}{cccc}
(1 / 2) B_{-} & 0 & 1 / 2 & 0 \\
(1 / 2) Z & (1 / 2) B_{+} & 0 & 0 \\
0 & 0 & B_{-} & 0 \\
0 & 0 & Z & B_{+}
\end{array}\right) \mathscr{\mathscr { H }}_{-}^{\mathscr{H}_{+}},
$$


where

$$
B=\left(\begin{array}{cc}
B_{-} & 0 \\
Z & B_{+}
\end{array}\right) \mathscr{\mathscr { H }}_{\overline{+}}^{\mathscr{H}_{+}} .
$$

It is immediate that $\sigma(T)=\mathbf{D}^{-}, \sigma_{e}(T)=\{\lambda \in \mathbf{C}:|\lambda|=0,1 / 2$, or 1$\}$, $\operatorname{ind}(\lambda-T)=-2$ for $0<|\lambda|<1 / 2$, and $\operatorname{ind}(\lambda-T)=-1$ for $1 / 2<|\lambda|<1$. (So that $T \notin \mathscr{W}^{-}$!)

A cumbersome calculation shows that $T$ is a $C_{00}$-construction with (isometrically isomorphic) $H^{\infty}(\mathbf{D})$ functional calculus, and $\mathscr{A}^{\prime}(T)=\mathscr{A}(T)$.

The proof of Proposition 5.1 is now complete.

Example 5.5 is a particular case of a large family of operators satisfying the condition $\mathscr{A}^{\prime}(T)=\mathscr{A}(T)$, to be described in a sequel article. Unfortunately, this family is not large enough to completely characterize $\left\{T \in \mathscr{L}(\mathscr{H}): \mathscr{A}^{\prime}(T)\right.$ $=\mathscr{A}(T)\}^{-}$.

\section{REFERENCES}

1. C. Apostol, The correction by compact perturbations of the singular behavior of operators, Rev. Roumaine Math. Pures Appl. 21 (1976), 155-175.

2. C. Apostol, L. A. Fialkow, D. A. Herrero, and D. Voiculescu, Approximation of Hilbert space operators, Volume II, Research Notes in Math., vol. 102, Pitman, Boston-LondonMelbourne, 1984.

3. C. Apostol, C. M. Pearcy, and N. Salinas, Spectra of compact perturbations of operators, Indiana Univ. Math. J. 26 (1977), 345-350.

4. S. R. Caradus, W. E. Pfaffenberger and B. Yood, Calkin algebras and algebras of operators on Banach spaces, Lecture Notes in Pure and Appl. Math., vol. 9, Marcel Dekker, New York, 1974.

5. T. W. Gamelin, Uniform algebras, Prentice Hall, Englewood Cliffs, N.J., 1969.

6. R. Gellar and D. A. Herrero, Hyperinvariant subspaces of bilateral weighted shifts, Indiana Univ. Math. J. 23 (1974), 771-790.

7. G. M. Goluzin, Geometric theory of functions of a complex variable, Amer. Math. Soc., Providence, R.I., 1969. (Translated from the Russian)

8. D. A. Herrero, A Rota universal model for operators with multiply connected spectrum, Rev. Roumaine Math. Pures Appl. 21 (1976), 15-23.

9. __ On multicyclic operators, Integral Equations Operator Theory 1 (1978), 57-102.

10. __ Quasisimilar operators with different spectra, Acta Sci. Math. (Szeged) 41 (1979).

11. _ _ Approximation of Hilbert space operators, Volume I, Research Notes in Math., vol. 72, Pitman, Boston-London-Melbourne, 1982.

12. _ The Fredholm structure of an n-multicyclic operator, Indiana Univ. Math. J. 36 (1987), 549-566.

13. _ Economical compact perturbations. II: Filling in the holes, J. Operator Theory 19 (1988), 25-42.

14. _ Similarity and approximation of operators, Proc. Sympos. Pure Math., vol. 51, part 1, Amer. Math. Soc., Providence, R.I., 1990, pp. 225-237.

15. _ A metatheorem on similarity and approximation of operators, J. London Math. Soc. (to appear).

16. D. A. Herrero and C.-L. Jiang, Limits of strongly irreducible operators and the Riesz decomposition theorem, Michigan J. Math. 37 (1990), 283-291. 
17. D. A. Herrero and N. Salinas, Analytically invariant and bi-invariant subspaces, Trans. Amer. Math. Soc. 173 (1972), 117-136.

18. T. Kato, Perturbation theory for nullity, deficiency, and other quantities of linear operators, J. Analyse Math. 6 (1958), 261-322.

19. __ Perturbation theory for linear operators, Springer-Verlag, New York, 1966.

20. G.-C. Rota, On models for linear operators, Comm. Pure Appl. Math. 13 (1960), 469-472.

21. R. Schatten, Norm ideals of completely continuous operators, Ergeb. Math. Grenzgeb., vol. 27, 2nd ed., Springer-Verlag, Berlin-Heidelberg-New York, 1970.

22. A. L. Shields and L. J. Wallen, The commutant of certain Hilbert space operators, Indiana Univ. Math. J. 20 (1971), 777-788.

23. B. Sz.-Nagy and C. Foiaş, Analyse, harmonique des opérateurs de l'espace de Hilbert, Akademiai Kaidó, Budapest and Masson, Paris, 1967.

24. D. Voiculescu, Norm-limits of algebraic operators, Rev. Roumaine Math. Pures Appl. 19 (1974), 371-378.

25. W. R. Wogen, On cyclicity of commutants, Integral Equations Operator Theory 5 (1982), 141-143.

Department of Mïathematics, Arizona State University, Tempe, Arizona 85287-1804 